\title{
$M^{a} \vee$ e phenomena

\section{A priori bounds and global bifurcation results for frequency combs modeled by the Lugiato-Lefever equation}

Rainer Mandel, Wolfgang Reichel

CRC Preprint 2016/7, March 2016

\section{KARLSRUHE INSTITUTE OF TECHNOLOGY}

\section{CRC 1173}

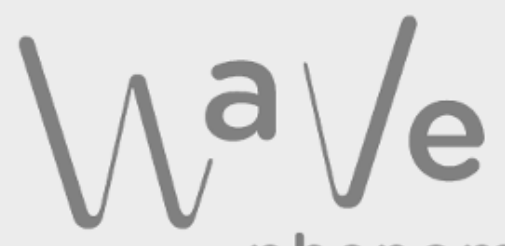

phenomena 


\section{Participating universities}

EBERHARD KARLS

UNIVERSITATT TUBINGEN

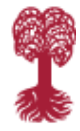

Funded by

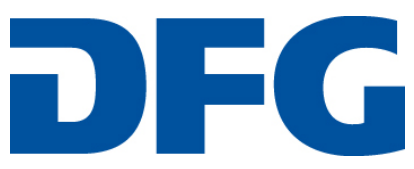

ISSN 2365-662X 


\title{
A PRIORI BOUNDS AND GLOBAL BIFURCATION RESULTS FOR FREQUENCY COMBS MODELED BY THE LUGIATO-LEFEVER EQUATION
}

\author{
RAINER MANDEL AND WOLFGANG REICHEL
}

\begin{abstract}
In nonlinear optics $2 \pi$-periodic solutions $a \in C^{2}([0,2 \pi] ; \mathbb{C})$ of the stationary Lugiato-Lefever equation $-d a^{\prime \prime}=(\mathrm{i}-\zeta) a+|a|^{2} a$ - if serve as a model for frequency combs, which are optical signals consisting of a superposition of modes with equally spaced frequencies. In accordance with experimental data we prove that nontrivial frequency combs can only be observed for special values of the forcing and detuning parameters $f, \zeta$. E.g., if the detuning parameter $\zeta$ is too large then nontrivial frequency combs do not exist, cf. Theorem 2. Additionally, we show that for large ranges of parameter values nontrivial frequency combs may be found on continua which bifurcate from curves of trivial frequency combs. Our results rely on the proof of a priori bounds for the stationary Lugiato-Lefever equation as well as a detailed rigorous bifurcation analysis based on the bifurcation theorems of Crandall-Rabinowitz and Rabinowitz.
\end{abstract}

\section{INTRODUCTION}

In physics literature an optical signal is called a frequency comb if it consists of a superposition of modes with equally spaced frequencies. By a suitable choice of reference frame a frequency comb becomes stationary (time-independent). For $k \in \mathbb{Z}$ let $\hat{a}_{k}$ denote the complex amplitude of the $k$-th mode of the signal in the frequency domain and let $a(x)=\sum_{k \in \mathbb{Z}} \hat{a}_{k} e^{i k x}$ be the associated Fourier series. A commonly used mathematical model is given by the stationary Lugiato-Lefever equation

$$
\begin{gathered}
-d a_{1}^{\prime \prime}=-a_{2}-\zeta a_{1}+\left(a_{1}^{2}+a_{2}^{2}\right) a_{1}, \\
-d a_{2}^{\prime \prime}=a_{1}-\zeta a_{2}+\left(a_{1}^{2}+a_{2}^{2}\right) a_{2}-f, \\
a_{1}, a_{2} 2 \pi \text {-periodic }
\end{gathered}
$$

where the parameters satisfy $d \neq 0$ and $\zeta, f \in \mathbb{R}$ and $a(x, t)=a_{1}(x)+\mathrm{i} a_{2}(x)$, i.e., $a$ is split into its real and imaginary part. Derivations of (1.1) may be found, e.g., in 2, 10]. More details on the physical background and the meaning of the parameters are given in Section 1.2 . Equation (1.1) is a nonvariational version of the stationary nonlinear Schrödinger equation with added damping and forcing. Our analysis and our results are based on the investigation of (1.1) from the point of view of bifurcation from trivial (i.e. spatially constant) solutions.

Date: March 18, 2016.

2000 Mathematics Subject Classification. Primary: 34C23, 34B15; Secondary: 35Q55, 35 Q60.

Key words and phrases. nonlinear Schrödinger equation, frequency combs, damping, forcing, bifurcation, Lugiato-Lefever equation. 
1.1. Mathematical context and main results. One first notices that (1.1) has trivial, i.e. spatially constant, solutions. They correspond to vanishing amplitudes of all modes except the 0-mode and are described in detail in Lemma 5 below. Stable, spatially periodic patterns bifurcating from trivial solutions of (1.1) were already observed in [16]. Recently, a more far-reaching bifurcation analysis appeared in [7], where the differential equation in (1.1) is considered as a four-dimensional dynamical system in the unknowns $a_{1}, a_{1}^{\prime}, a_{2}, a_{2}^{\prime}$. As the parameters $f$ and $\zeta$ change, the trivial solutions exhibit various bifurcation phenomena. This approach allows an extensive account of various possible types of solutions of the differential equations in (1.1). However, with this approach the $2 \pi$-periodicity of the solutions may be lost. An emanating solution with spatial period $\tau$ has to be rescaled to the fixed period $2 \pi$ and as a result (1.1) is changed, e.g., $d$ becomes $(2 \pi)^{2} d / \tau^{2}$. A different view on bifurcation has been developed in [17]. Here spatially $2 \pi$-periodic solutions of (1.1) are considered via a bifurcation approach using the center manifold reduction. The resulting picture is very detailed in the vicinity of special parameter values but beyond these values nothing seems to be known - a gap in the literature which we would like to fill with the present paper.

Similarly to the above-mentioned papers we use bifurcation theory to prove the existence of frequency combs bifurcating from the set of spatially constant solutions. Let us therefore point out the main features which distinguish our approach from the previous ones. Unlike [7] we consider (1.1) on certain spaces of $2 \pi$-periodic functions. We obtain a very rich bifurcation picture which is not limited to local considerations as in [7, 17]. In Theorem 1 and Theorem 2 we find a priori bounds and uniqueness results which allow us to show that

(a) nonconstant solutions of $(1.1)$ only occur in the range $\operatorname{sign}(d) \zeta \in\left[\zeta_{*}, \zeta^{*}\right]$,

(b) nonconstant solutions of (1.1) satisfy $\|a\|_{\infty}+|\zeta| \leq C$.

Here, the values $\zeta_{*}, \zeta^{*}$ and $C$ are explicit and only depend on the parameters $f, d$. We begin with our results concerning pointwise a priori bounds for solutions of (1.1) in terms of the parameters $\zeta, d, f$.

Theorem 1. Let $d \neq 0, f, \zeta \in \mathbb{R}$. Every solution $a \in C^{2}\left([0,2 \pi], \mathbb{R}^{2}\right)$ of (1.1) satisfies

$$
\|a\|_{L^{\infty}} \leq \frac{|f|\left(1+12 \pi^{2} f^{2}|d|^{-1}\right)}{\max \{1,-\zeta \operatorname{sign}(d)-\gamma(d, f)\}}
$$

where

$$
\gamma(d, f):= \begin{cases}36 \pi^{2} f^{4}|d|^{-1}, & d>0 \\ 36 \pi^{2} f^{4}|d|^{-1}+f^{2}\left(1+12 \pi^{2} f^{2}|d|^{-1}\right)^{2}, & d<0 .\end{cases}
$$

Remark. Further bounds in $L^{2}\left([0,2 \pi], \mathbb{R}^{2}\right)$ and $H^{1}\left([0,2 \pi], \mathbb{R}^{2}\right)$ may be extracted from the proof of this theorem.

In our second result we employ Theorem 1 to show that the set of nonconstant solutions is bounded with respect to $\zeta$.

Theorem 2. Let $d \neq 0, f \in \mathbb{R}, \zeta \in \mathbb{R}$ and let $\zeta_{*}, \zeta^{*}$ be given by

$$
\begin{aligned}
\zeta_{*} & :=-\gamma(d, f)-\sqrt{6}|f|\left(1+12 \pi^{2} f^{2}|d|^{-1}\right), \\
\zeta^{*} & :=6 f^{2}\left(1+12 \pi^{2} f^{2}|d|^{-1}\right)^{2} .
\end{aligned}
$$


Then every solution of (1.1) is constant provided $\operatorname{sign}(d) \zeta<\zeta_{*}$ or $\operatorname{sign}(d) \zeta>\zeta^{*}$.

Next we consider (1.1) from the point of view of bifurcation theory where one of the two values $f$ or $\zeta$ is fixed and the other one is the bifurcation parameter. According to the two possible choices we identify two curves of constant solutions: $\hat{\Gamma}_{f}$ with $f$ being fixed and $\bar{\Gamma}_{\zeta}$ with $\zeta$ fixed, see Lemma 5 for explicit parametrizations of these curves. We investigate branches of nontrivial solutions that bifurcate from the trivial branches $\hat{\Gamma}_{f}$ and $\bar{\Gamma}_{\zeta}$ and obtain information about their global shape. In our approach we consider the following special class of solutions of (1.1). We call them synchronized solutions in order to emphasize that they have a particular shape.

Definition. $A 2 \pi$-periodic solution $a \in C^{2}([0,2 \pi] ; \mathbb{C})$ of $(1.1)$ is called synchronized if $a^{\prime}(0)=$ $a^{\prime}(\pi)=0$.

Synchronized solutions are even around $x=0$ and $x=\pi$. The advantage of considering synchronized solutions is that the translation invariance of the original equation $(1.1)$ is no longer present in this Neumann boundary value problem which makes the bifurcation analysis much easier, see also the remark after Proposition 10.

Before we state our results let us recall some common notions in bifurcation theory. In the context of bifurcation from $\hat{\Gamma}_{f}$ (Theorem 3 a pair $(a, \zeta)$ is called a trivial solution if it is spatially constant, i.e., if $(a, \zeta)$ lies on $\hat{\Gamma}_{f}$. A trivial solution $(a, \zeta)$ is called a bifurcation point if a sequence $\left(a_{k}, \zeta_{k}\right)_{k \in \mathbb{N}}$ of non-trivial solutions of the periodic system (1.1) converges to $(a, \zeta)$. Similarly trivial solutions and bifurcation points are defined when bifurcation from $\bar{\Gamma}_{\zeta}$ is investigated, see Theorem 4. Since our analysis of synchronized solutions is based on the bifurcation theorem of Crandall-Rabinowitz [4] such bifurcating non-trivial solutions lie on local curves around the bifurcation points. Furthermore, we will use the global bifurcation theorem of Rabinowitz [19] to show that these curves are part of a connected set that is unbounded or returns to the curve of trivial solutions at some other point. A continuum satisfying one of these two properties will be a called global continuum. If, additionally, the nontrivial (i.e. nonconstant) solutions from this continuum are confined in a bounded subset of $L^{\infty}\left([0,2 \pi], \mathbb{R}^{2}\right) \times \mathbb{R}$ then the continuum will be called bounded.

For fixed $f \in \mathbb{R}$ we find that at most finitely many global continua bifurcate from $\hat{\Gamma}_{f}$. These continua are bounded and intersect $\hat{\Gamma}_{f}$ at another trivial solution. For the reader's convenience this is illustrated in the plots of Figures 9 14. The result reads as follows.

Theorem 3. Let $d \neq 0, f \in \mathbb{R}$. If $|f|<1$ then the curve $\hat{\Gamma}_{f}$ does not contain any bifurcation point for (1.1). In case $|f| \geq 1$ the following holds:

(i) All bifurcation points are among the points $\left(\hat{a}_{1}(t), \hat{a}_{2}(t), \hat{\zeta}(t)\right)$ where the number $t \in$ $\left[-\sqrt{1-|f|^{-2}},+\sqrt{1-|f|^{-2}}\right]$ satisfies

$$
d k^{2}=f^{2}\left(1-t^{2}\right)-\frac{t}{\sqrt{1-t^{2}}}-\sigma \sqrt{f^{4}\left(1-t^{2}\right)^{2}-1}
$$

for some $k \in \mathbb{N}$ and some $\sigma \in\{-1,1\}$. 
(ii) The curve $\hat{\Gamma}_{f}$ contains at most $\hat{k}(f)$ bifurcation points for 1.1 where

$$
\hat{k}(f):=2\left(|d|^{-1}\left(f^{2}+\sqrt{f^{2}-1}+\sqrt{f^{4}-1}\right)\right)^{1 / 2} .
$$

(iii) If in addition to (1.4) one has

(S) $-k^{2}+2 d^{-1}\left(f^{2}\left(1-t^{2}\right)-t\left(1-t^{2}\right)^{-1 / 2}\right) \neq j^{2}$ for all $j \in \mathbb{N}_{0} \backslash\{k\}$,

(T) $4 f^{6} t^{3}\left(1-t^{2}\right)^{2}+f^{4}\left(1-t^{2}\right)^{1 / 2}-2 t f^{2}-\left(1-t^{2}\right)^{-3 / 2}$

$$
-\sigma \sqrt{f^{4}\left(1-t^{2}\right)^{2}-1}\left(4 f^{4} t^{3}\left(1-t^{2}\right)+f^{2}\left(2 t^{2}-1\right)\left(1-t^{2}\right)^{-1 / 2}\right) \neq 0
$$

then a global continuum containing nontrivial synchronized solutions emanates from $\left(\hat{a}_{1}(t), \hat{a}_{2}(t), \hat{\zeta}(t)\right)$. This continuum is bounded and it returns to $\hat{\Gamma}_{f}$ at some other point.

Remark. The statement that emanating branches must return to the family of constant solutions at some other point follows from Rabinowitz' global bifurcation theorem [19] and the fact that all nonconstant solutions $(a, \zeta)$ of (1.1) satisfy $\|a\|_{\infty}+|\zeta| \leq C$ for some positive number $C$ depending only the parameters $f, d$, cf. Theorem 1 and 2 .

Now let us state the corresponding result for the bifurcation analysis associated to the family of trivial solutions $\bar{\Gamma}_{\zeta}$ for given $\zeta \in \mathbb{R}$. In contrast to the above results we find that infinitely many global continua emanate from $\bar{\Gamma}_{\zeta}$ in the case $d>0$ whereas for $d<0$ again only finitely many such continua can exist. The numerical plots from Figures 18 illustrate our results. Although we do not have a proof for the boundedness of the continua in this case, it seems nevertheless plausible in view of the numerical plots.

Theorem 4. Let $d \neq 0, \zeta \in \mathbb{R}$.

(i) All bifurcation points on the curve $\bar{\Gamma}_{\zeta}$ are among $\left(\bar{a}_{1}(s), \bar{a}_{2}(s), \bar{f}(s)\right)$ where

$$
s=\sigma_{2}\left(\frac{2}{3}\left(\zeta+d k^{2}\right)-\frac{\sigma_{1}}{3} \sqrt{\left(\zeta+d k^{2}\right)^{2}-3}\right)^{1 / 2}
$$

for some $k \in \mathbb{N}$, some $\sigma_{1}, \sigma_{2} \in\{-1,1\}$ and provided $\zeta+d k^{2} \geq \sqrt{3}$.

(ii) If $d<0$ then the curve $\bar{\Gamma}_{\zeta}$ contains at most $\bar{k}(\zeta)$ bifurcation points for (1.1) where

$$
\bar{k}(\zeta):=4\left(|d|^{-1}(\zeta-\sqrt{3})_{+}\right)^{1 / 2}
$$

(iii) If in addition to (1.5) one has

(S) $-k^{2}+\frac{2}{3} d^{-1}\left(\zeta+4 d k^{2}-2 \sigma_{1} \sqrt{\left(\zeta+d k^{2}\right)^{2}-3}\right) \neq j^{2}$ for all $j \in \mathbb{N}_{0} \backslash\{k\}$,

(T) $\zeta+d k^{2} \neq \sqrt{3}$ and $4 \zeta+d k^{2}-2 \sigma_{1} \sqrt{\left(\zeta+d k^{2}\right)^{2}-3} \neq 0$ and

$$
2 \zeta+5 d k^{2}-4 \sigma_{1} \sqrt{\left(\zeta+d k^{2}\right)^{2}-3} \neq 0
$$

then a global continuum containing synchronized nontrivial solutions bifurcates from $\left(\bar{a}_{1}(s), \bar{a}_{2}(s), \bar{f}(s)\right)$.

Remarks. (1) In principle the exceptional points where the conditions $(\mathrm{S})$ and $(\mathrm{T})$ are not satisfied could be analyzed using different bifurcation theorems. Suitable candidates for a bifurcation theorem in the presence of higher-dimensional kernels are the theorems of Healey, Kielhöfer, Krömer [14] and Westreich [21]. Bifurcation results without transversality condition can be found in [15]. However, the required amount of calculations are far too 
high to justify the use of these theorems. For the same reason we did not include a detailed analysis of the initial directions of the bifurcation branches which, without any theoretical difficulty, may be calculated using the formulas from section I.6 in Kielhöfer's book [12.

(2) It would be interesting to find out whether (1.1) admits solutions which are not synchronized. Note that in the case of scalar periodic boundary value problems the restriction to homogeneous Neumann boundary conditions on an interval of half the period is natural since (up to a shift) all solutions satisfy this condition. However, in the system case this is not clear at all.

1.2. Frequency combs in physics and engineering. Currently frequency combs are gaining interest as optical sources for high-speed data transmission where the individual comb lines are used as carriers. A high power per combline with the same spectral power distribution is important. An experimental set-up for such frequency combs is given by a microresonator which is coupled to an optical waveguide under the influence of a single, strong, external laser source that is tuned to a resonance wavelength of the device. Inside the resonator the optical intensity is strongly enhanced and modes start to interact in a nonlinear way. As a consequence, the primarily excited mode couples with a multitude of neighboring modes. This leads to a cascaded transfer of power from the pump to the comb lines. Under suitable choice of parameters, a stationary cascade of excited modes can be obtained and results in a stable frequency comb with equidistant spectral lines. If $\hat{a}_{k}(t)$ denotes the dimensionless complex-valued amplitude of the $k$-th mode in the microresonator at time $t$ then, following [2,10], it satisfies the following set of coupled differential equations

$$
\mathrm{i} \partial_{t} \hat{a}_{k}(t)=(-\mathrm{i}+\zeta) \hat{a}_{k}(t)+d k^{2} \hat{a}_{k}(t)-\sum_{k^{\prime}, k^{\prime \prime} \in \mathbb{Z}} \hat{a}_{k^{\prime}}(t) \hat{a}_{k^{\prime \prime}}(t) \overline{\hat{a}}_{k^{\prime}+k^{\prime \prime}-k}(t)+\mathrm{i} \delta_{0 k} f, \quad k \in \mathbb{Z} .
$$

In this equation, the parameters $\zeta, d, f$ are real and $t$ is normalized time. The term $\mathrm{i} \delta_{0 k} f$ corresponds to forcing by the external pump, $\zeta$ represents the normalized frequency detuning between the source and the principal resonance of the microresonator, and $d$ quantifies the dispersion in the system. The case $d<0$ corresponds to normal dispersion whereas $d>0$ is called the anomalous regime, cf. [7]. The loss of power due to radiation and waveguide coupling is modeled by the damping term $-\mathrm{i} \hat{a}_{k}(t)$. In the literature a stationary solution of (1.6) is called a frequency comb.

Via the Fourier series $a(x, t)=\sum_{k \in \mathbb{Z}} \hat{a}_{k}(t) e^{\mathrm{i} k x}$ frequency combs can equally be defined as stationary solutions of the Lugiato-Lefever equation

$$
\mathrm{i} \partial_{t} a(x, t)=(-\mathrm{i}+\zeta) a(x, t)-d \partial_{x}^{2} a(x, t)-|a(x, t)|^{2} a(x, t)+\mathrm{i} f, \quad x \in \mathbb{R} / 2 \pi \mathbb{Z}, t \in \mathbb{R} .
$$

It was originally proposed in [16] as a model for the envelope of a field transmitted through a nonlinearly responding optical cavity. It resembles a nonlinear Schrödinger equation with added damping and forcing. Stationary solutions of (1.7) of the form $a=a_{1}+\mathrm{i} a_{2}$ correspond to solutions $\left(a_{1}, a_{2}\right)$ of $(1.1)$.

The experimental generation of frequency combs in microresonators has been demonstrated many times, cf. the review paper [13]. One of the first demonstrations [5] used a toroidal 
fused-silica microresonator. In [10] the dynamics of the Kerr comb formation process is experimentally explored and found to be independent of the resonator material system and geometry. One of the first theoretical papers [2] marks the starting point for a series of subsequent investigations and publications. In this paper a numerical simulation of Kerr frequency combs is given based on (1.6), i.e., the modal expansion of the fields. A considerable computational effort is needed to handle the multitude of coupled differential equations. Nevertheless, a detailed account of the temporal dynamics is supplied and analytical expressions (approximations) of the distance of primary comb lines in terms of resonator and pump parameters are derived.

As one can see in Section 5 there a many different shapes of frequency combs. Of particular interest are so-called soliton combs. These are stationary solutions of (1.7) which are highly localized in space. Accordingly their frequency spectrum shows many densely spaced comb lines; cf. Figures 8 and 14 . Moreover, the power of the $k$-th excited frequency in a soliton comb is much higher than the $k$-th excited frequency in a comb with sparse frequency spectrum, cf. Figure 3 and 4 . Since these properties of soliton combs are very desirable for high-speed data transmission, they received attention in recent literature. In [3] a numerical study of pump and resonator parameters and their effect on the bandwidth of Kerr combs was performed, and first indications appeared that soliton combs can only be achieved by a special tuning of the pump parameters. According to the simulations presented in [6] these solitons show a high coherence along with a high number of comb lines with flat power distribution. The first experimental proof of soliton combs was done in [9]. The effect of higher order dispersion terms is the topic of [18]. It is shown that incorporating third-order dispersion terms into the model enlarges the parameter ranges where stable soliton combs exist. In [20] the effect of higher order dispersion on the comb shape is discussed.

1.3. Further mathematical results. A rigorous study of the time-dependent problem (1.7) both from the analytical and from the numerical point of view was recently given in [11]. Applying Theorem 2.1 of [11] to the function $a(x, t) e^{\mathrm{i} \zeta t}$ one obtains that for $d=1$ and initial data lying in $H_{\text {per }}^{4}([0,2 \pi] ; \overline{\mathbb{C}})$ the initial value problem associated to (1.7) admits a unique solution

$$
a \in C\left(\mathbb{R}_{+} ; H_{\text {per }}^{4}([0,2 \pi] ; \mathbb{C})\right) \cap C^{1}\left(\mathbb{R}_{+} ; H_{\text {per }}^{2}([0,2 \pi] ; \mathbb{C})\right) \cap C^{2}\left(\mathbb{R}_{+} ; L_{\text {per }}^{2}([0,2 \pi] ; \mathbb{C})\right)
$$

satisfying the additional bounds $\|a(t)\|_{2} \leq C,\|a(t)\|_{H^{1}} \leq C \sqrt{1+t}$ for some positive number $C>0$ which is independent of $t$. Furthermore, the paper provides a detailed analysis of the Strang splitting associated to 1.7 including error bounds in $L_{\text {per }}^{2}([0,2 \pi] ; \mathbb{C}), H_{\text {per }}^{1}([0,2 \pi] ; \mathbb{C})$ as well as estimates related to the stability properties of the numerical scheme. Further numerical and analytical results related to periodically forced and damped NLS may be found in $[1,8]$.

1.4. Structure of the paper. The paper is organized as follows. In Section 2 we provide the functional analytical framework for our analysis. This includes an appropriate choice of the function spaces and corresponding solution concepts. In Section 3 the proofs of Theorem 1 (a priori bounds) and Theorem 2 (uniqueness) are given. The proofs of the bifurcation results 
from Theorem 3 and 4 can be found in Section 4 . The final Section 5 contains illustrations with tables of bifurcation points, bifurcation diagrams and plots of approximate solutions.

1.5. On the generation of the numerical plots. The illustrations in Section 5 were created with the software package AUTO. It is a free software which determines bifurcation points, approximations of solutions and generates bifurcation diagrams. It can be downloaded from indy.cs.concordia.ca/auto/. We postprocessed the outupt of AUTO by a MATLAB program to improve the quality of the approximated solutions of (1.1) via several Newton iterations and to compute the Fourier coefficients of the improved approximated solutions. The MATLAB program also produces .pdf files of plotted solutions and their Fourier coefficients. A .zip file containing a README-description and driver files for the code can be downloaded freely from www.waves.kit.edu/downloads/CRC1173_Preprint_2016-7_supplement.zip. By running the driver files AUTO and MATLAB will be invoked and generate all plots of this paper.

\section{Mathematical Setup}

First we describe the spaces of solutions in which our analysis works. A weak solution $a \in H^{1}\left([0,2 \pi] ; \mathbb{R}^{2}\right)$ of $[1.1$ will be called a solution for the sake of simplicity. Notice that every such solution coincides almost everywhere with a smooth classical solution of the equation so that regularity issues will not play a role in the sequel and all solution concepts in fact coincide. In the context of Theorems 1 and 2 it is convenient to consider $2 \pi$-periodic classical solutions. For the proof of Theorem 2 the space $H_{\text {per }}^{2}\left([0,2 \pi] ; \mathbb{R}^{2}\right)$ will be useful. In the context of the bifurcation results of Theorems 3 and 4 we consider synchronized solutions of (1.1), i.e. solutions that satisfy additionally $a^{\prime}(0)=a^{\prime}(\pi)=0$. If we set $H:=H^{1}\left([0, \pi], \mathbb{R}^{2}\right)$ then $a=\left(a_{1}, a_{2}\right)$ is a synchronized solution of $[1.1)$ if and only if $a=\left(a_{1}, a_{2}\right) \in H$ satisfies

$$
\begin{array}{ll}
\int_{0}^{\pi} d a_{1}^{\prime} \varphi_{1}^{\prime} d x=\int_{0}^{\pi}\left(-a_{2}-\zeta a_{1}+\left(a_{1}^{2}+a_{2}^{2}\right) a_{1}\right) \varphi_{1} d x & \text { for all } \varphi_{1} \in H, \\
\int_{0}^{\pi} d a_{2}^{\prime} \varphi_{2}^{\prime} d x=\int_{0}^{\pi}\left(a_{1}-\zeta a_{2}+\left(a_{1}^{2}+a_{2}^{2}\right) a_{2}-f\right) \varphi_{2} d x & \text { for all } \varphi_{2} \in H .
\end{array}
$$

A synchronized solution can be extended evenly around $x=\pi$ and thus produce a $2 \pi$-periodic function. This weak setting in the Hilbert space $H$ will be convenient for the proof of the bifurcation results.

Next we describe the trivial (i.e. spatially constant) solutions of (1.1). In order to obtain a global parameterization of the solution curves some new auxiliary parameters $t$ resp. $s$ will be used instead of $\zeta$ resp. $f$. The totality of constant solutions is given next.

Lemma 5. Let $d \neq 0$ be fixed.

(a) Let $f \in \mathbb{R}$ be given. Then the set of constant solutions $\left(a_{1}, a_{2}, \zeta\right)$ of (1.1) is given by $\hat{\Gamma}_{f}=\left\{\left(\hat{a}_{1}(t), \hat{a}_{2}(t), \hat{\zeta}(t)\right):|t|<1\right\}$ where

$$
\hat{a}_{1}(t)=f\left(1-t^{2}\right), \quad \hat{a}_{2}(t)=-f t \sqrt{1-t^{2}}, \quad \hat{\zeta}(t)=f^{2}\left(1-t^{2}\right)+\frac{t}{\sqrt{1-t^{2}}} .
$$


(b) Let $\zeta \in \mathbb{R}$ be given. Then the set of constant solutions $\left(a_{1}, a_{2}, f\right)$ of $(1.1)$ is given by $\bar{\Gamma}_{\zeta}=\left\{\left(\bar{a}_{1}(s), \bar{a}_{2}(s), \bar{f}(s)\right): s \in \mathbb{R}\right\}$ where

$$
\bar{a}_{1}(s)=\frac{s}{\sqrt{1+\left(s^{2}-\zeta\right)^{2}}}, \quad \bar{a}_{2}(s)=\frac{s\left(s^{2}-\zeta\right)}{\sqrt{1+\left(s^{2}-\zeta\right)^{2}}}, \quad \bar{f}(s)=s \sqrt{1+\left(s^{2}-\zeta\right)^{2}} .
$$

Proof. Let us first show that every constant solution $a=\left(a_{1}, a_{2}\right) \in \mathbb{R}^{2} \subset H$ of (1.1) satisfies

$$
f^{2}=|a|^{2}\left(1+\left(|a|^{2}-\zeta\right)^{2}\right) .
$$

Indeed, for constant solutions (1.1) can be written as

$$
\left(\begin{array}{cc}
|a|^{2}-\zeta & -1 \\
1 & |a|^{2}-\zeta
\end{array}\right)\left(\begin{array}{l}
a_{1} \\
a_{2}
\end{array}\right)=\left(\begin{array}{l}
0 \\
f
\end{array}\right)
$$

and hence by inverting the matrix

$$
\left(\begin{array}{l}
a_{1} \\
a_{2}
\end{array}\right)=\frac{f}{1+\left(|a|^{2}-\zeta\right)^{2}}\left(\begin{array}{c}
1 \\
|a|^{2}-\zeta
\end{array}\right)
$$

Taking the Euclidean norm on both sides of the equation gives 2.1.

Now let us prove (a), so let $f \in \mathbb{R}$ be given and define $t \in(-1,1)$ via $t\left(1-t^{2}\right)^{-1 / 2}=\zeta-|a|^{2}$. Then (2.1) implies

$$
\zeta-t\left(1-t^{2}\right)^{-1 / 2}=|a|^{2}=\frac{f^{2}}{1+t^{2}\left(1-t^{2}\right)^{-1}}=f^{2}\left(1-t^{2}\right)
$$

and hence

$$
\zeta=f^{2}\left(1-t^{2}\right)+\frac{t}{\sqrt{1-t^{2}}}
$$

From the linear system (2.2) and the definition of $t$ we obtain the desired formulas for $a_{1}, a_{2}$.

In order to prove (b) let $\zeta \in \mathbb{R}$ and set $s:=\operatorname{sign}(f)|a|$. Then we have

$$
f^{2}=s^{2}\left(1+\left(s^{2}-\zeta\right)^{2}\right), \quad \text { hence } f=s \sqrt{1+\left(s^{2}-\zeta\right)^{2}} .
$$

From the linear system $(2.2)$ and this formula for $s$ we obtain the result.

\section{Proof of Theorem 1 and Theorem 2}

We always assume $d \neq 0$ and $f, \zeta \in \mathbb{R}$. We write $\|\cdot\|_{p}$ for the standard norm on $L^{p}\left([0,2 \pi] ; \mathbb{R}^{2}\right)$ for $p \in[1, \infty]$.

Proof of Theorem 1: We divide the proof into several steps.

Step 1: Here we prove the $L^{2}$-estimate $\|a\|_{2} \leq \sqrt{2 \pi}|f|$. To this end we define the $2 \pi$-periodic function $g:[0,2 \pi] \rightarrow \mathbb{R}$ by

By using 1.1 one finds

$$
g:=d\left(a_{2} a_{1}^{\prime}-a_{1} a_{2}^{\prime}\right)^{\prime} .
$$

$$
g=d a_{2} a_{1}^{\prime \prime}-d a_{1} a_{2}^{\prime \prime}=a_{2}\left(a_{2}+\zeta a_{1}-|a|^{2} a_{1}\right)+a_{1}\left(a_{1}-\zeta a_{2}+|a|^{2} a_{2}-f\right)=|a|^{2}-f a_{1} .
$$


Since $a_{2} a_{1}^{\prime}-a_{1} a_{2}^{\prime}$ is $2 \pi$-periodic we obtain

$$
0=\int_{0}^{2 \pi} g d x=\int_{0}^{2 \pi}\left(|a|^{2}-f a_{1}\right) d x \geq\|a\|_{2}^{2}-\sqrt{2 \pi}|f|\|a\|_{2}
$$

which implies the desired $L^{2}$-bound

$$
\|a\|_{2} \leq \sqrt{2 \pi}|f|
$$

Step 2: Next we prove the estimate $|d|\left\|a^{\prime}\right\|_{2} \leq 6 \pi f^{2}\|a\|_{2}$ and thus $|d|\left\|a^{\prime}\right\|_{2} \leq 6 \sqrt{2} \pi^{3 / 2}|f|^{3}$ due to (3.2). Using the differential equation (1.1) we get

$$
\begin{aligned}
|d|\left\|a^{\prime}\right\|_{2}^{2} & =|d| \int_{0}^{2 \pi} a_{1}^{\prime}\left(-d a_{2}^{\prime \prime}+\zeta a_{2}-|a|^{2} a_{2}+f\right)^{\prime} d x+a_{2}^{\prime}\left(d a_{1}^{\prime \prime}-\zeta a_{1}+|a|^{2} a_{1}\right)^{\prime} d x \\
& =|d| d \int_{0}^{2 \pi}\left(a_{2}^{\prime} a_{1}^{\prime \prime \prime}-a_{1}^{\prime} a_{2}^{\prime \prime \prime}\right) d x+|d| \int_{0}^{2 \pi} a_{1}^{\prime}\left(-|a|^{2} a_{2}\right)^{\prime}+a_{2}^{\prime}\left(|a|^{2} a_{1}\right)^{\prime} d x \\
& =|d| d \int_{0}^{2 \pi}\left(a_{2}^{\prime} a_{1}^{\prime \prime}-a_{1}^{\prime} a_{2}^{\prime \prime}\right)^{\prime} d x+|d| \int_{0}^{2 \pi}\left(-a_{2} a_{1}^{\prime}+a_{1} a_{2}^{\prime}\right)\left(|a|^{2}\right)^{\prime} d x \\
& =0+|d| \int_{0}^{2 \pi}\left(a_{2} a_{1}^{\prime}-a_{1} a_{2}^{\prime}\right)^{\prime}|a|^{2} d x \\
& \leq \int_{0}^{2 \pi}|g||a|^{2} d x \\
& \leq\|g\|_{\infty}\|a\|_{2}^{2} \\
& \leq \sqrt{2 \pi}|f|\|g\|_{\infty}\|a\|_{2} .
\end{aligned}
$$

Note that $g$ is the derivative of a $2 \pi$-periodic function and therefore satisfies $\int_{0}^{2 \pi} g d x=0$. Hence there exists $x_{0} \in[0,2 \pi]$ such that $g\left(x_{0}\right)=0$ and the supremum norm of $g$ can be estimated as follows

$$
\begin{aligned}
\|g\|_{\infty} & \leq \sup _{x \in[0,2 \pi]}\left|g(x)-g\left(x_{0}\right)\right| \\
& \leq \int_{0}^{2 \pi}\left|g^{\prime}\right| d x \\
& \leq \int_{0}^{2 \pi} 2\left|a^{\prime}\right||a|+|f|\left|a_{1}^{\prime}\right| d x \\
& \leq\left(2\|a\|_{2}+\sqrt{2 \pi}|f|\right)\left\|a^{\prime}\right\|_{2} \\
& \leq 3 \sqrt{2 \pi}|f|\left\|a^{\prime}\right\|_{2} .
\end{aligned}
$$

By our previous estimates (3.3) and 3.2 this gives

$$
|d|\left\|a^{\prime}\right\|_{2} \leq \sqrt{2 \pi}|f|\|a\|_{2} \cdot 3 \sqrt{2 \pi}|f|=6 \pi f^{2}\|a\|_{2} \leq 6 \sqrt{2} \pi^{3 / 2}|f|^{3}
$$

which finishes step 2. 
Step 3: Now we show the first of two $L^{\infty}$-bounds: $\|a\|_{\infty} \leq|f|\left(1+12 \pi^{2} f^{2}|d|^{-1}\right)$. From the $L^{2}$-estimate (3.2) we infer that there is an $x_{1} \in I$ satisfying $\left|a\left(x_{1}\right)\right| \leq|f|$. Hence our first $L^{\infty}$-estimate follows from

$$
\begin{aligned}
\|a\|_{\infty} & \leq\left|a\left(x_{1}\right)\right|+\left\|a-a\left(x_{1}\right)\right\|_{\infty} \\
& \leq|f|+\left\|a^{\prime}\right\|_{1} \\
& \leq|f|+\sqrt{2 \pi}\left\|a^{\prime}\right\|_{2} \\
& \quad \leq|f|+\sqrt{2 \pi} \cdot 6 \sqrt{2} \pi^{3 / 2}|f|^{3}|d|^{-1} \\
& =|f|\left(1+12 \pi^{2} f^{2}|d|^{-1}\right) .
\end{aligned}
$$

Step 4: Next we show

$$
\|a\|_{2} \leq(-\zeta \operatorname{sign}(d)-\gamma(d, f))^{-1} \sqrt{2 \pi}|f| .
$$

whenever $-\zeta \operatorname{sign}(d)-\gamma(d, f)>0$ for $\gamma(d, f)$ from (1.3). Testing (1.1) with $\left(a_{1}, a_{2}\right)$ and adding up the resulting equations yields

$$
d\left\|a^{\prime}\right\|_{2}^{2}=-\zeta\|a\|_{2}^{2}+\|a\|_{4}^{4}-f \int_{0}^{2 \pi} a_{2} d x .
$$

This can be used in the following way.

$$
\begin{aligned}
36 \pi^{2} f^{4}|d|^{-1}\|a\|_{2}^{2} & =|d|^{-1}\left(6 \pi f^{2}\|a\|_{2}\right)^{2} \\
& \stackrel{\sqrt[3.4]{\geq}}{\geq}|d|^{-1}\left(|d|\left\|a^{\prime}\right\|_{2}\right)^{2} \\
& =|d|\left\|a^{\prime}\right\|_{2}^{2} \\
& \stackrel{3.7}{=}-\zeta \operatorname{sign}(d)\|a\|_{2}^{2}+\operatorname{sign}(d)\|a\|_{4}^{4}-\operatorname{sign}(d) f \int_{0}^{2 \pi} a_{2} d x .
\end{aligned}
$$

In order to prove $(3.6)$ we first suppose $d>0$. Then $(3.8)$ implies

$$
36 \pi^{2} f^{4}|d|^{-1}\|a\|_{2}^{2} \geq-\zeta\|a\|_{2}^{2}-\sqrt{2 \pi}|f|\|a\|_{2}
$$

from which we infer the desired bound

$$
\sqrt{2 \pi}|f| \geq\left(-\zeta-36 \pi^{2} f^{4}|d|^{-1}\right)\|a\|_{2}=(-\zeta-\gamma(d, f))\|a\|_{2} .
$$

Supposing now $d<0$ we find that $(3.8)$ implies

$$
\begin{aligned}
36 \pi^{2} f^{4}|d|^{-1}\|a\|_{2}^{2} & \geq\left(\zeta-\|a\|_{\infty}^{2}\right)\|a\|_{2}^{2}-\sqrt{2 \pi}|f|\|a\|_{2} \\
& \stackrel{3}{\geq}\left(\zeta-f^{2}\left(1+12 \pi^{2} f^{2}|d|^{-1}\right)^{2}\right)\|a\|_{2}^{2}-\sqrt{2 \pi}|f|\|a\|_{2}
\end{aligned}
$$

from which we obtain

$$
\sqrt{2 \pi}|f| \geq\left(\zeta-36 \pi^{2} f^{4}|d|^{-1}-f^{2}\left(1+12 \pi^{2} f^{2}|d|^{-1}\right)^{2}\|a\|_{2}=(\zeta-\gamma(d, f))\|a\|_{2} .\right.
$$

so that 3.6 is proved. 
Step 5: Finally, we show

$$
\|a\|_{\infty} \leq(-\zeta \operatorname{sign}(d)-\gamma(d, f))^{-1}|f|\left(1+12 \pi^{2} f^{2}|d|^{-1}\right) .
$$

$-\zeta \operatorname{sign}(d)-\gamma(d, f)>0$. This completes the proof of Theorem 1. The $L^{2}$-estimate from Step 4 entails, just as in Step 3, that there exists some $x_{1} \in[0,2 \pi]$ such that $\left|a\left(x_{1}\right)\right| \leq$ $(-\zeta \operatorname{sign}(d)-\gamma(d, f))^{-1}|f|$ and the claim follows from

$$
\begin{aligned}
\|a\|_{\infty} & \leq\left|a\left(x_{1}\right)\right|+\sqrt{2 \pi}\left\|a^{\prime}\right\|_{2} \\
& \stackrel{\text { 3.4 }}{\leq}\left|a\left(x_{1}\right)\right|+6 \pi \sqrt{2 \pi} f^{2}|d|^{-1}\|a\|_{2} \\
& \leq \frac{|f|\left(1+12 \pi^{2} f^{2}|d|^{-1}\right)}{-\zeta \operatorname{sign}(d)-\gamma(d, f)} \quad \text { by Step } 4 .
\end{aligned}
$$

Now we come to the proof of the uniqueness result from Theorem 2. Let us first outline our strategy to prove the result. We use the fact that a solution $a=\left(a_{1}, a_{2}\right):[0,2 \pi] \rightarrow \mathbb{R} \times \mathbb{R}$ of (1.1) is constant if and only if the function $A=\left(A_{1}, A_{2}\right):=\left(a_{1}^{\prime}, a_{2}^{\prime}\right)$ is trivial. Since $\left(a_{1}, a_{2}\right)$ solves (1.1) the functions $A_{1}, A_{2}$ satisfy the boundary value problem

$$
\begin{gathered}
-d A_{1}^{\prime \prime}=-A_{2}-\zeta A_{1}+\left(3 a_{1}^{2}+a_{2}^{2}\right) A_{1}+2 a_{1} a_{2} A_{2}, \\
-d A_{2}^{\prime \prime}=A_{1}-\zeta A_{2}+2 a_{1} a_{2} A_{1}+\left(a_{1}^{2}+3 a_{2}^{2}\right) A_{2}, \\
A_{1}, A_{2} 2 \pi \text {-periodic. }
\end{gathered}
$$

In view of 3.9$)$ it is natural to study the operator

$$
L_{d, \zeta}:\left\{\begin{aligned}
H_{\mathrm{per}}^{2}\left([0,2 \pi] ; \mathbb{R}^{2}\right) & \rightarrow L^{2}\left([0,2 \pi] ; \mathbb{R}^{2}\right), \\
\left(B_{1}, B_{2}\right) & \mapsto\left(-d B_{1}^{\prime \prime}+\zeta B_{1}+B_{2},-d B_{2}^{\prime \prime}+\zeta B_{2}-B_{1}\right) .
\end{aligned}\right.
$$

Using the fact that the embedding Id : $H_{\text {per }}^{2}\left([0,2 \pi] ; \mathbb{R}^{2}\right) \rightarrow L^{2}\left([0,2 \pi] ; \mathbb{R}^{2}\right)$ is compact we obtain the following result.

Lemma 6. The operator $L_{d, \zeta}$ has a bounded inverse $L_{d, \zeta}^{-1}: L^{2}\left([0,2 \pi] ; \mathbb{R}^{2}\right) \rightarrow H_{\mathrm{per}}^{2}\left([0,2 \pi] ; \mathbb{R}^{2}\right)$ with the property that $\operatorname{Id} \circ L_{d, \zeta}^{-1}: L^{2}\left([0,2 \pi] ; \mathbb{R}^{2}\right) \rightarrow L^{2}\left([0,2 \pi] ; \mathbb{R}^{2}\right)$ is compact and $\left\|\operatorname{Id} \circ L_{d, \zeta}^{-1}\right\| \leq$ $\min \left\{1,(\operatorname{sign}(d) \zeta)_{+}^{-1}\right\}$.

Proof. Let $\left(B_{1}, B_{2}\right) \in H_{\text {per }}^{2}\left([0,2 \pi] ; \mathbb{R}^{2}\right)$ and $\left(g_{1}, g_{2}\right) \in L^{2}\left([0,2 \pi] ; \mathbb{R}^{2}\right)$ satisfy $L_{d, \zeta}\left(B_{1}, B_{2}\right)=$ $\left(g_{1}, g_{2}\right)$, i.e., $-d B_{1}^{\prime \prime}+\zeta B_{2}+B_{2}=g_{1}$ and $-d B_{2}^{\prime \prime}+\zeta B_{2}-B_{1}=g_{2}$. Testing these differential equations with $\left(B_{1}, B_{2}\right)$ respectively $\left(B_{2},-B_{1}\right)$ and adding up the resulting equations yields

$$
\begin{aligned}
d\left(\left\|B_{1}^{\prime}\right\|_{2}^{2}+\left\|B_{2}^{\prime}\right\|_{2}^{2}\right)+\zeta\left(\left\|B_{1}\right\|_{2}^{2}+\left\|B_{2}\right\|_{2}^{2}\right) & =\int_{0}^{2 \pi}\left(g_{1} B_{1}+g_{2} B_{2}\right) d x, \\
\left\|B_{1}\right\|_{2}^{2}+\left\|B_{2}\right\|_{2}^{2} & =\int_{0}^{2 \pi}\left(g_{1} B_{2}-g_{2} B_{1}\right) d x .
\end{aligned}
$$

The second line (3.11) shows that $L_{d, \zeta}$ is injective. Moreover, using $\left\|B_{1}^{\prime}\right\|_{2}^{2}+\left\|B_{2}^{\prime}\right\|_{2}^{2} \geq 0$ as well as Hölder's inequality we obtain from (3.10) and (3.11)

$$
\max \{1, \operatorname{sign}(d) \zeta\}\left\|\left(B_{1}, B_{2}\right)\right\|_{2}^{2} \leq\left\|\left(g_{1}, g_{2}\right)\right\|_{2}\left\|\left(B_{1}, B_{2}\right)\right\|_{2}
$$


and thus

$$
\left\|\left(B_{1}, B_{2}\right)\right\|_{2} \leq \min \left\{1,(\operatorname{sign}(d) \zeta)_{+}^{-1}\right\}\left\|\left(g_{1}, g_{2}\right)\right\|_{2} .
$$

From this and 3.10$)$ we get the estimate

$$
|d|\left\|\left(B_{1}^{\prime}, B_{2}^{\prime}\right)\right\|_{2}^{2} \leq(1+|\zeta|)\left\|\left(g_{1}, g_{2}\right)\right\|_{2}^{2}
$$

and using the differential equation and the $L^{2}$-estimate on $\left(B_{1}, B_{2}\right)$ we find

$$
|d|\left\|\left(B_{1}^{\prime \prime}, B_{2}^{\prime \prime}\right)\right\|_{2} \leq(2+|\zeta|)\left\|\left(g_{1}, g_{2}\right)\right\|_{2} .
$$

This proves the bounded invertibility of $L_{d, \zeta}$ as well as the norm estimate for $\operatorname{Id} \circ L_{d, \zeta}^{-1}$.

Proof of Theorem 2: If $\left(A_{1}, A_{2}\right)$ satisfies (3.9) then we have $\left(A_{1}, A_{2}\right)=K_{a}\left(A_{1}, A_{2}\right)$ where $K_{a}: L^{2}\left([0,2 \pi] ; \mathbb{R}^{2}\right) \rightarrow L^{2}\left([0,2 \pi] ; \mathbb{R}^{2}\right)$ is given by

$$
K_{a}\left(\begin{array}{l}
A_{1} \\
A_{2}
\end{array}\right):=\operatorname{Id} \circ L_{d, \zeta}^{-1}\left(M_{a}\left(\begin{array}{l}
A_{1} \\
A_{2}
\end{array}\right)\right) \text { with } M_{a}=\left(\begin{array}{cc}
3 a_{1}^{2}+a_{2}^{2} & 2 a_{1} a_{2} \\
2 a_{1} a_{2} & a_{1}^{2}+3 a_{2}^{2}
\end{array}\right) .
$$

Hence, it suffices to prove that the operator norm $\left\|K_{a}\right\|$ is less than 1 whenever $\operatorname{sign}(d) \zeta>\zeta^{*}$ or $\operatorname{sign}(d) \zeta<\zeta_{*}$. Consider the matrix $M_{a}$ as a map from $L^{2}\left([0,2 \pi] ; \mathbb{R}^{2}\right)$ into itself. Then its operator norm is bounded as follows

$$
\left\|M_{a}\right\| \leq\left\|\left(\begin{array}{cc}
4\|a\|_{\infty}^{2} & 2\|a\|_{\infty}^{2} \\
2\|a\|_{\infty}^{2} & 4\|a\|_{\infty}^{2}
\end{array}\right)\right\|=\|a\|_{\infty}^{2} \cdot\left\|\left(\begin{array}{cc}
4 & 2 \\
2 & 4
\end{array}\right)\right\|=6\|a\|_{\infty}^{2}
$$

since the largest eigenvalue of $\left(\begin{array}{ll}4 & 2 \\ 2 & 4\end{array}\right)$ is 6 . Combining this inequality with the estimate from Lemma 6 we get

$$
\left\|K_{a}\right\| \leq 6 \min \left\{1,(\operatorname{sign}(d) \zeta)_{+}^{-1}\right\}\|a\|_{\infty}^{2} .
$$

In the first case, where $\operatorname{sign}(d) \zeta>\zeta^{*}>0$, Theorem 1 gives, by choice of $\zeta^{*}$,

$$
\left\|K_{a}\right\| \leq 6(\operatorname{sign}(d) \zeta)^{-1}\|a\|_{\infty}^{2}<6\left(\zeta^{*}\right)^{-1} f^{2}\left(1+12 \pi^{2} f^{2}|d|^{-1}\right)^{2}=1
$$

In the second case, where $\operatorname{sign}(d) \zeta<\zeta_{*}<0$ and in particular $-\zeta \operatorname{sign}(d)-\gamma(d, f)>0$, we get from Theorem 1 and again by the choice of $\zeta_{*}$

$$
\left\|K_{a}\right\| \leq 6\|a\|_{\infty}^{2} \leq\left(\frac{\sqrt{6}|f|\left(1+12 \pi^{2} f^{4}|d|^{-1}\right)}{-\zeta \operatorname{sign}(d)-\gamma(d, f)}\right)^{2}<\left(\frac{\sqrt{6}|f|\left(1+12 \pi^{2} f^{4}|d|^{-1}\right)}{-\zeta_{*}-\gamma(d, f)}\right)^{2}=1
$$

which is all we had to show.

\section{Proof of Theorem 3 and Theorem 4}

In this section we prove the bifurcation results for the Lugiato-Lefever equation (1.1). We will always assume that $d \neq 0$ is fixed. As explained earlier our sufficient conditions for bifurcation from constant solutions of (1.1) will be established in the context of the Neumann boundary value problem

$$
\begin{array}{ll}
-d a_{1}^{\prime \prime}=-a_{2}-\zeta a_{1}+\left(a_{1}^{2}+a_{2}^{2}\right) a_{1}, & a_{1}^{\prime}(0)=a_{1}^{\prime}(\pi)=0, \\
-d a_{2}^{\prime \prime}=a_{1}-\zeta a_{2}+\left(a_{1}^{2}+a_{2}^{2}\right) a_{2}-f, & a_{2}^{\prime}(0)=a_{2}^{\prime}(\pi)=0 .
\end{array}
$$


In this way the shift-invariance of the general $2 \pi$-periodic system is circumvented. Using the notation introduced in the introduction we will find the existence of synchronized solution branches bifurcating from the curves of constant solutions.

Let us now shortly outline how our bifurcation analysis is organized. In Section 4.1 we first provide a functional analytical framework for solutions of (4.1). The construction of solutions is done with the help of the bifurcation theorem due to Crandall and Rabinowitz (Theorem 7). In Theorem 3 , the family of trivial solutions will be $\hat{\Gamma}_{f}$ for fixed $f \in \mathbb{R}$ and in Theorem 4 it will be $\bar{\Gamma}_{\zeta}$ for fixed $\zeta \in \mathbb{R}$. The proof of these theorems is accomplished in four steps. In Section 4.2 we first determine the candidates for the bifurcation points of (1.1) with respect to $\hat{\Gamma}_{f}, \bar{\Gamma}_{\zeta}$ proving Theorem 3(i), Theorem 4(i). This result will be used in Section 4.3 to establish the upper bounds for the number of bifurcation points claimed in Theorem 3(ii) and Theorem 4(ii). In order to prove the existence of bifurcating branches it remains to check the hypotheses of the Crandall-Rabinowitz theorem in the context of the Neumann boundary value problem (4.1). In Section 4.4 we show that the kernels at the possible bifurcation points (calculated in Section 4.2) are simple if the conditions (S) from the respective theorem holds. In the same way the transversality condition will be verified in Section 4.5 supposing that condition $(\mathrm{T})$ holds. Hence, a direct application of Theorem 7 establishes the existence of local curves containing nontrivial solutions of (4.1) that emanate from $\hat{\Gamma}_{f}, \bar{\Gamma}_{\zeta}$ respectively. Moreover, the a priori bounds for $a$ from Theorem 1 and the uniqueness result from Theorem 2 tell us that for any given $f \in \mathbb{R}$ the continua emanating from $\hat{\Gamma}_{f}$ must be bounded with respect to both variables $a, \zeta$ so that Rabinowitz' global bifurcation theorem [19] yields that each continuum returns to $\hat{\Gamma}_{f}$ at another point. Hence, part (iii) of Theorem 3 and Theorem 4 is shown and the proof is complete.

\subsection{Functional analytical framework and preliminaries.}

We look for solutions of 4.1 in the function space $H:=H^{1}\left([0, \pi] ; \mathbb{R}^{2}\right)$. It is a Hilbert space with the inner product $\langle\cdot, \cdot\rangle_{H}$ given by

$$
\langle\varphi, \psi\rangle_{H}:=\int_{0}^{\pi}|d|\left(\varphi_{1}^{\prime} \psi_{1}^{\prime}+\varphi_{2}^{\prime} \psi_{2}^{\prime}\right)+\varphi_{1} \psi_{1}+\varphi_{2} \psi_{2} d x \quad \text { for } \varphi=\left(\begin{array}{c}
\varphi_{1} \\
\varphi_{2}
\end{array}\right), \psi=\left(\begin{array}{c}
\psi_{1} \\
\psi_{2}
\end{array}\right) \in H .
$$

If $D: \operatorname{dom}(D) \rightarrow \mathbb{R}$ denotes the selfadjoint realization of the differential operator $\varphi \mapsto$ $-|d| \varphi^{\prime \prime}+\varphi$ with homogeneous Neumann boundary values at $0, \pi$ then $\langle\varphi, \psi\rangle_{H}=\langle D \varphi, \psi\rangle_{L^{2}}$ for all $\varphi \in \operatorname{dom}(D)$. Even though it will not be used in the sequel let us state without proof

$$
\operatorname{dom}(D)=\left\{\varphi \in H^{2}\left([0, \pi] ; \mathbb{R}^{2}\right): \varphi^{\prime}(0)=\varphi^{\prime}(\pi)=\left(\begin{array}{l}
0 \\
0
\end{array}\right)\right\}
$$

The operator $D$ has a compact inverse $D^{-1}: H \rightarrow \operatorname{dom}(D) \subset H$ so that (4.1) may be rewritten as $G(a, \zeta, f)=0$ where the function $G: H \times \mathbb{R} \times \mathbb{R} \rightarrow H$ is given by

$$
G(a, \zeta, f):=\operatorname{sign}(d) a-D^{-1}\left(-\zeta a+\operatorname{sign}(d) a+|a|^{2} a+\left(\begin{array}{c}
-a_{2} \\
a_{1}
\end{array}\right)+\left(\begin{array}{c}
0 \\
-f
\end{array}\right)\right) .
$$

In order to prove bifurcation results from the family of constant solutions of (4.1) let us recall the Crandall-Rabinowitz bifurcation theorem. 
Theorem 7 (Crandall-Rabinowitz [4]). Let $I \subset \mathbb{R}$ be an open interval and let $F: H \times I \rightarrow H$ be twice continuously differentiable such that $F(0, \lambda)=0$ for all $\lambda \in I$ and such that $F_{x}\left(0, \lambda_{0}\right)$ is an index-zero Fredholm operator for $\lambda_{0} \in I$. Moreover assume:

(H1) there is $\varphi \in H, \varphi \neq 0$ such that $\operatorname{ker}\left(F_{x}\left(0, \lambda_{0}\right)\right)=\operatorname{span}\{\varphi\}$,

(H2) $\left\langle F_{x \lambda}\left(0, \lambda_{0}\right)[\varphi], \varphi^{*}\right\rangle_{H} \neq 0$ where $\operatorname{ker}\left(F_{x}\left(0, \lambda_{0}\right)^{*}\right)=\operatorname{span}\left\{\varphi^{*}\right\}$.

Then there exists $\epsilon>0$ and a continuously differentiable curve $(x, \lambda):(-\epsilon, \epsilon) \rightarrow H \times \mathbb{R}$ with $\lambda(0)=\lambda_{0}, x(0)=0, x^{\prime}(0)=\varphi$ and $x(t) \neq 0$ for $0<|t|<\epsilon$ and $F(x(t), \lambda(t))=0$ for all $t \in(-\epsilon, \epsilon)$. Moreover, there exists a neighbourhood $U \times J \subset H \times I$ of $\left(0, \lambda_{0}\right)$ such that all nontrivial solutions in $U \times J$ of $F(x, \lambda)=0$ lie on the curve.

In the proof of Theorem 3 and Theorem 4 we will apply Theorem 7 to the functions $\hat{F}: H \times(-1,1) \rightarrow H$ and $\bar{F}: H \times \mathbb{R} \rightarrow H$ given by

$$
\begin{array}{ll}
\hat{F}(b, t) & :=G(b+\hat{a}(t), \hat{\zeta}(t), f), \quad b \in H, t \in(-1,1), \\
\bar{F}(b, s) & :=G(b+\bar{a}(s), \zeta, \bar{f}(s)), \quad b \in H, s \in \mathbb{R}
\end{array}
$$

where the trivial solution curves $(\hat{a}(t), \hat{\zeta}(t))$ respectively $(\bar{a}(s), \zeta(s))$ are taken from Lemma 5 . Checking the assumptions of Theorem 7 requires the calculation of the derivatives of $\hat{F}, \bar{F}$ at the trivial solutions. The necessary preparations are made in the following proposition.

Proposition 8. Let $a \in H, \zeta, f \in \mathbb{R}$ and let the $2 \times 2$-matrices $N(a, \zeta), M_{1}(a), M_{2}(a)$ be given by

$$
N(a, \zeta)=\left(\begin{array}{cc}
-\zeta+3 a_{1}^{2}+a_{2}^{2} & -1+2 a_{1} a_{2} \\
1+2 a_{1} a_{2} & -\zeta+a_{1}^{2}+3 a_{2}^{2}
\end{array}\right), M_{1}(a)=\left(\begin{array}{cc}
6 a_{1} & 2 a_{2} \\
2 a_{2} & 2 a_{1}
\end{array}\right), M_{2}(a)=\left(\begin{array}{cc}
2 a_{2} & 2 a_{1} \\
2 a_{1} & 6 a_{2}
\end{array}\right) .
$$

Then we have for all $\varphi, \psi \in H$

$$
\begin{aligned}
G_{a}(a, \zeta, f)[\varphi] & =\operatorname{sign}(d) \varphi-D^{-1}(N(a, \zeta) \varphi+\operatorname{sign}(d) \varphi), \\
G_{a a}(a, \zeta, f)[\varphi, \psi] & =-D^{-1}\left(\begin{array}{l}
\varphi^{T} M_{1}(a) \psi \\
\varphi^{T} M_{2}(a) \psi
\end{array}\right)
\end{aligned}
$$

as well as

$$
G_{a \zeta}(a, \zeta, f)[\varphi]=D^{-1} \varphi, \quad G_{a f}(a, \zeta, f)[\varphi]=0, \quad G_{\zeta}(a, \zeta, f)=D^{-1} a, \quad G_{f}(a, f)=D^{-1}\left(\begin{array}{l}
0 \\
1
\end{array}\right)
$$

The proof of Proposition 8 is mere calculation and will therefore be dropped. In the next proposition we characterize $\operatorname{ker}\left(G_{a}(a, \zeta, f)\right)$ and $\operatorname{ker}\left(G_{a}(a, \zeta, f)^{*}\right)$ at a constant solution $a \in \mathbb{R}^{2} \subset H$ of 4.1 .

Proposition 9. Let $\zeta, f \in \mathbb{R}$. Then for every constant solution $a=\left(a_{1}, a_{2}\right) \in \mathbb{R}^{2} \subset H$ of (4.1) we have

$$
\begin{aligned}
\operatorname{ker}\left(G_{a}(a, \zeta, f)\right) & \left.=\operatorname{span}\left\{\varphi_{k}(a): k \in \mathbb{N}_{0} \text { satisfies } 4.6\right)\right\} \\
\operatorname{ker}\left(G_{a}(a, \zeta, f)^{*}\right) & \left.=\operatorname{span}\left\{\varphi_{k}^{*}(a): k \in \mathbb{N}_{0} \text { satisfies } 4.6\right)\right\}
\end{aligned}
$$

where

$$
\left(\zeta+d k^{2}\right)^{2}-4|a|^{2}\left(\zeta+d k^{2}\right)+1+3|a|^{4}=0
$$


and

$$
\begin{aligned}
& \varphi_{k}(a)(x)=\alpha^{k} \cos (k x), \quad \varphi_{k}^{*}(a)(x)=\beta^{k} \cos (k x) \quad \text { with } \alpha^{k}, \beta^{k} \in \mathbb{R}^{2} \text { given by } \\
& \alpha^{k}=\left\{\begin{array}{c}
\left(\begin{array}{c}
1-2 a_{1} a_{2} \\
3 a_{1}^{2}+a_{2}^{2}-\zeta-d k^{2}
\end{array}\right) \quad \text { if } a_{1} a_{2} \neq \frac{1}{2} \quad \text { or } 3 a_{1}^{2}+a_{2}^{2} \neq \zeta+d k^{2}, \\
a_{1}^{2}+3 a_{2}^{2}-\zeta-d k^{2} \\
-1-2 a_{1} a_{2}
\end{array}\right) \quad \text { if } a_{1} a_{2}=\frac{1}{2} \quad \text { and } 3 a_{1}^{2}+a_{2}^{2}=\zeta+d k^{2}, \\
& \beta^{k}=\left\{\begin{array}{c}
\left(\begin{array}{c}
-1-2 a_{1} a_{2} \\
3 a_{1}^{2}+a_{2}^{2}-\zeta-d k^{2} \\
a_{1}^{2}+3 a_{2}^{2}-\zeta-d k^{2} \\
1-2 a_{1} a_{2}
\end{array}\right) \quad \text { if } a_{1} a_{2} \neq-\frac{1}{2} \text { or } 3 a_{1}^{2}+a_{2}^{2} \neq \zeta+d k^{2}, \\
\text { if } a_{1} a_{2}=-\frac{1}{2} \quad \text { and } 3 a_{1}^{2}+a_{2}^{2}=\zeta+d k^{2} .
\end{array}\right.
\end{aligned}
$$

Proof. By Proposition 8 every function $\varphi \in \operatorname{ker}\left(G_{a}(a, \zeta, f)\right)$ satisfies $-d \varphi^{\prime \prime}=N(a, \zeta) \varphi$ in $(0, \pi)$ and $\varphi^{\prime}(0)=\varphi^{\prime}(\pi)=0$. From the Fourier series expansion

$$
\varphi(x)=\sum_{k \in \mathbb{N}_{0}} \alpha^{k} \cos (k x) \quad \text { for } x \in[0, \pi]
$$

we obtain the equation

$$
\sum_{k \in \mathbb{N}_{0}}\left(d k^{2} \operatorname{Id}-N(a, \zeta)\right) \alpha^{k} \cos (k x)=0 \quad \text { for } x \in[0, \pi] .
$$

Hence, for all $k \in \mathbb{N}_{0}$ the vector $\alpha^{k}$ lies in the kernel of the matrix

$$
d k^{2} \operatorname{Id}-N(a, \zeta)=\left(\begin{array}{cc}
\zeta+d k^{2}-3 a_{1}^{2}-a_{2}^{2} & 1-2 a_{1} a_{2} \\
-1-2 a_{1} a_{2} & \zeta+d k^{2}-a_{1}^{2}-3 a_{2}^{2}
\end{array}\right) .
$$

This implies that $\operatorname{ker}\left(G_{a}(a, \zeta, f)\right)$ is nontrivial if and only if the determinant of one of these matrices vanishes. Calculating $\operatorname{det}\left(d k^{2} \mathrm{Id}-N(a, \zeta)\right)$ for all $k \in \mathbb{N}_{0}$ we obtain that $\operatorname{ker}\left(G_{a}(a, \zeta, f)\right)$ is nontrivial if and only if there is a solution $k \in \mathbb{N}_{0}$ of (4.6). In that case the kernel of $d k^{2} \operatorname{Id}-N(a, \zeta)$ is spanned by the vector $\varphi_{k}(a)$ given by 4.7) which proves the formula for $\operatorname{ker}\left(G_{a}(a, \zeta, f)\right)$ from (4.4). A similar calculation shows that $\varphi^{*} \in \operatorname{ker}\left(G_{a}(a, \zeta, f)^{*}\right)$ satisfies $-d \varphi^{* \prime \prime}=N(a, \zeta)^{T} \varphi^{*}$ in $(0, \pi)$ and $\varphi^{* \prime}(0)=\varphi^{* \prime}(\pi)=0$. From this the formula 4.5 for $\operatorname{ker}\left(G_{a}(a, \zeta, f)^{*}\right)$ follows as above.

Since for every given $\zeta, f \in \mathbb{R}$ equation (4.6) has at most two different solutions $k_{1}, k_{2} \in \mathbb{N}_{0}$ we know that the spaces $\operatorname{ker}\left(G_{a}(a, \zeta, f)\right)$ are at most two-dimensional. In the following proposition we single out those parameters for which we have one-dimensional kernels.

Proposition 10. Let $\zeta, f, a$ be chosen as in Proposition 9 such that (4.6) holds for some $k \in \mathbb{N}_{0}$. Then $\operatorname{ker}\left(G_{a}(a, \zeta, f)\right)$ and $\operatorname{ker}\left(G_{a}(a, \zeta, f)^{*}\right)$ are one-dimensional if and only if

$$
-k^{2}+d^{-1}\left(4|a|^{2}-2 \zeta\right) \neq j^{2} \quad \text { for all } j \in \mathbb{N}_{0} \backslash\{k\} \text {. }
$$


Proof. Let $\operatorname{ker}\left(G_{a}(a, \zeta, f)\right)$ contain two linearly independent nontrivial vectors. Proposition9 then implies that equation (4.6) has a second solution $j \in \mathbb{N}_{0}$ which gives

$$
\left(\zeta+d k^{2}\right)^{2}-4\left(\zeta+d k^{2}\right)|a|^{2}=\left(\zeta+d j^{2}\right)^{2}-4\left(\zeta+d j^{2}\right)|a|^{2} \text { and } \zeta+d k^{2} \neq \zeta+d j^{2} .
$$

From this we infer $2 \zeta+d k^{2}+d j^{2}=4|a|^{2}$ or equivalently

$$
-k^{2}+d^{-1}\left(4|a|^{2}-2 \zeta\right)=j^{2} \quad \text { for some } j \in \mathbb{N}_{0} \backslash\{k\} .
$$

Vice versa, by (4.4), this condition implies that $\operatorname{ker}\left(G_{a}(a, \zeta, f)\right)$ is two-dimensional and the result follows.

Remark. The applicability of the Crandall-Rabinowitz Theorem relies on the simplicity of the kernel of the linearized equation, which we will check using Proposition 10. In the setting of $2 \pi$-periodic functions simplicity of the kernel of the linearized equation never holds. This can be seen as follows: First notice that (4.6), which is a necessary condition for bifurcation for the Neumann problem (4.1), is also a necessary condition for bifurcation for the $2 \pi$-periodic problem (1.1). The proof from above only needs small changes: the operator $D \varphi:=\left(-|d| \varphi_{1}^{\prime \prime}+\right.$ $\left.\varphi_{1},-|d| \varphi_{2}^{\prime \prime}+\varphi_{2}\right)$, now equipped with periodic boundary conditions on $[0,2 \pi]$, has a compact inverse $D^{-1}: H_{\text {per }} \rightarrow H_{\text {per }}$ where $H_{\text {per }}$ denotes the restriction of $2 \pi$-periodic functions from $H^{1}\left(\mathbb{R} ; \mathbb{R}^{2}\right)$ to the interval $(0,2 \pi)$. Furthermore, in the Fourier series expansion 4.9$)$ the terms $\tilde{\alpha}^{k} \sin (k x)$ with vectors $\tilde{\alpha}^{k} \in \mathbb{R}^{2}$ additionally occur. The vanishing of $\operatorname{det}\left(d k^{2} \operatorname{Id}-N(a, \zeta)\right)$ then appears in the same way as a necessary condition for the nontrivial solvability of the linear equation $-d \varphi^{\prime \prime}=N(a, \zeta) \varphi$ by a $2 \pi$-periodic function $\varphi$. However, with $a^{k} \cos (k x)$ belonging to the $\operatorname{ker}\left(G_{a}(a, \zeta, f)\right)$ for some $k \in \mathbb{N}$ also $a^{k} \sin (k x)$ belongs to the kernel making it at least two-dimensional. This is one of the reasons why we chose to consider synchronized solutions rather than periodic solutions.

\subsection{Determination of all possible bifurcation points.}

First let us mention that the solutions of (4.6) for $k=0$ do not give rise to bifurcation from $\hat{\Gamma}_{f}, \bar{\Gamma}_{\zeta}$ regardless of whether the assumptions (S),(T) are satisfied. This is not in contradiction with the Crandall-Rabinowitz Theorem for the following reason. In our analysis we use the parameterizations of $\hat{\Gamma}_{f}, \bar{\Gamma}_{\zeta}$ from Lemma 5 having the property that $t \mapsto \hat{\zeta}(t), s \mapsto \bar{f}(s)$ may not be injective for some parameter samples. In our bifurcation analysis related to $k=0$ this inconvenience leads to a false prediction of bifurcation in the following way. In order to keep the explanations short we explain the situation only for the bifurcation analysis related to $\hat{\Gamma}_{f}$. Since we use $t$ (and not $\zeta$ ) as the parameter in the Crandall-Rabinowitz theorem we find a bifurcating branch w.r.t. $t$ whenever $(4.6)$ as well as $(\mathrm{S})$ and $(\mathrm{T})$ are satisfied for some $k \in \mathbb{N}_{0}$ and some $t_{0} \in(-1,1)$. One can check that in the special case $k=0$ this is equivalent to saying that the curve $\hat{\zeta}$ has a turning point at $t_{0}$, i.e. we have $\hat{\zeta}^{\prime}\left(t_{0}\right)=0, \hat{\zeta}^{\prime \prime}\left(t_{0}\right) \neq 0$. As a consequence, for any given $\varepsilon$ close enough to 0 there is a value $\delta_{\varepsilon}$ converging to 0 as $\varepsilon \rightarrow 0$ such that $\delta_{\varepsilon} \cdot \varepsilon<0$ and $\hat{\zeta}\left(t_{0}+\varepsilon\right)=\hat{\zeta}\left(t_{0}+\delta_{\varepsilon}\right)$. Hence the bifurcation theorem detects the branch $\left(t_{0}+\varepsilon, \hat{a}\left(t_{0}+\delta_{\varepsilon}\right)\right)$ bifurcating from $\left(t_{0}+\epsilon, \hat{a}\left(t_{0}+\epsilon\right)\right)$ at $\epsilon=0$. Clearly, $\left(\hat{\zeta}\left(t_{0}+\varepsilon\right), \hat{a}\left(t_{0}+\delta_{\varepsilon}\right)\right)=\left(\hat{\zeta}\left(t_{0}+\delta_{\varepsilon}\right), \hat{a}\left(t_{0}+\delta_{\varepsilon}\right)\right)$ still lies on $\hat{\Gamma}_{f}$ and so this branch bifurcates with respect to the variable $t$, but not with respect to the variable $\zeta$. 
For that reason the case $k=0$ will be left aside when we determine the possible bifurcation points. Note that this phenomenon could be avoided if we locally parameterized the trivial solution families $\hat{\Gamma}_{f}$ by $\zeta$. However, since this parameterization is in general not global further technical complications would arise.

In Theorem 3: For given $f \in \mathbb{R}$ we have to determine all $t \in(-1,1)$ such that $\hat{F}_{a}(0, t)=$ $G_{a}(\hat{a}(t), \hat{\zeta}(t), f)$ has a nontrivial kernel. According to Proposition 9 this is the case if and only if there is $k \in \mathbb{N}_{0}$ such that

$$
\left(\hat{\zeta}(t)+d k^{2}-2|\hat{a}(t)|^{2}\right)^{2}=|\hat{a}(t)|^{4}-1 .
$$

In particular this implies $1 \leq|\hat{a}(t)|^{2}=f^{2}\left(1-t^{2}\right.$ ) (see Lemma 5) so that $|f| \geq 1$ is a necessary condition for bifurcation from $\hat{\Gamma}_{f}$. Furthermore, in case $|f| \geq 1$, we obtain from the formulas for $\hat{a}(t), \hat{\zeta}(t)$ (see Lemma 5 ) and (4.10)

$$
|t| \leq 1-|f|^{-2} \quad \text { and } \quad d k^{2}=f^{2}\left(1-t^{2}\right)-\frac{t}{\sqrt{1-t^{2}}}-\sigma \sqrt{f^{4}\left(1-t^{2}\right)^{2}-1}
$$

for some $\sigma \in\{-1,1\}$ so that part (i) of Theorem 3 is proved.

In Theorem 4: Now let $\zeta \in \mathbb{R}$ be fixed. Proposition 9 and $|\bar{a}(s)|^{2}=s^{2}$ imply that the operator $\bar{F}_{a}(0, s)=G_{a}(\bar{a}(s), \zeta, \bar{f}(s))$ has a nontrivial kernel if and only if

$$
\left(\zeta+d k^{2}-2 s^{2}\right)^{2}=s^{4}-1 .
$$

This implies $\left(\zeta+d k^{2}\right)^{2} \geq 3$ and $s^{2}=\frac{2}{3}\left(\zeta+d k^{2}\right)-\frac{\sigma_{1}}{3}\left(\left(\zeta+d k^{2}\right)^{2}-3\right)^{1 / 2}$ for some $\sigma_{1} \in\{ \pm 1\}$. From the nonnegativity of $s^{2}$ we infer $\zeta+d k^{2} \geq 0$ and thus $\zeta+d k^{2} \geq \sqrt{3}$. Hence, $s$ is given by the formula (1.5) for $\sigma_{1}, \sigma_{2} \in\{-1,1\}$ and part (i) of Theorem 4 is proved.

\subsection{Number of bifurcation points.}

In Theorem 3: We have to prove that for all $f \in \mathbb{R}$ the trivial solution family $\hat{\Gamma}_{f}$ contains at most $\hat{k}(f)$ bifurcation points where $\hat{k}(f)$ was defined in Theorem 3 (ii). By Proposition 9 every bifurcation point $(a, \zeta) \in \hat{\Gamma}_{f}$ satisfies the quadratic equation 4.6 for some $k \in \mathbb{N}$. Hence every $k$ gives rise to at most two bifurcation points and therefore it suffices to prove $2 k \leq \hat{k}(f)$. Formula 4.6 implies

$$
0 \leq\left(\zeta+d k^{2}-2|a|^{2}\right)^{2}=|a|^{4}-1 .
$$

This shows that bifurcation can only occur if $|a| \geq 1$ and together with (2.1) from the Appendix we get

$$
f^{2} \geq|a|^{2} \quad \text { and } \quad f^{2} \geq 1+\left(|a|^{2}-\zeta\right)^{2} .
$$

Substituting $\zeta$ from (4.6) we obtain $\zeta+d k^{2}-2|a|^{2}= \pm \sqrt{|a|^{4}-1}$ and thus

$$
\begin{aligned}
|d| k^{2} & \leq\left.\left.\left|d k^{2}-\right| a\right|^{2} \mp \sqrt{|a|^{4}-1}|+| a\right|^{2}+\sqrt{|a|^{4}-1} \\
& =\left.|| a\right|^{2}-\zeta||+|a|^{2}+\sqrt{|a|^{4}-1} \\
& \leq \sqrt{f^{2}-1}+f^{2}+\sqrt{f^{4}-1} .
\end{aligned}
$$

From this inequality we directly conclude $2 k \leq \hat{k}(f)$. 
In Theorem 4: Let $\zeta \in \mathbb{R}$. Arguing as above we find that every bifurcation point $(a, \zeta) \in \bar{\Gamma}_{f}$ satisfies equation (1.5) for some $k \in \mathbb{N}, \sigma_{1}, \sigma_{2} \in\{-1,1\}$ and hence gives rise to at most four bifurcation points. Therefore we have to prove $4 k \leq \bar{k}(\zeta)$ in case $d<0$. Indeed, in that case the inequality $\zeta+d k^{2} \geq \sqrt{3}$ from $(1.5)$ implies $k \leq\left(|d|^{-1}(\zeta-\sqrt{3})_{+}\right)^{1 / 2}$ which is all we had to show.

\subsection{Simplicity of the kernels.}

In Theorem 3: Let $f \in \mathbb{R}$ and let $(\hat{a}(t), \hat{\zeta}(t))$ be a possible bifurcation point with respect to $\hat{\Gamma}_{f}$, i.e., we assume that $t \in(-1,1)$ satisfies equation 1.4$)$ for some $k \in \mathbb{N}$ and some $\sigma \in\{-1,1\}$. Then Proposition 10 implies that $\operatorname{ker}\left(\hat{F}_{a}(0, t)\right)$ is one-dimensional if and only if we have

$$
-k^{2}+d^{-1}\left(4|\hat{a}(t)|^{2}-2 \hat{\zeta}(t)\right) \notin\left(\mathbb{N}_{0} \backslash\{k\}\right)^{2} .
$$

Since we know from Lemma 5 (a) that

$$
4|\hat{a}(t)|^{2}-2 \hat{\zeta}(t)=2 f^{2}\left(1-t^{2}\right)-2 t\left(1-t^{2}\right)^{-1 / 2}
$$

4.11) is guaranteed by condition (S) of Theorem 3 (iii) and we are done.

In Theorem 4: Let $\zeta \in \mathbb{R}$ and let $(\bar{a}(s), \bar{f}(s))$ be a possible bifurcation point with respect to $\bar{\Gamma}_{\zeta}$, i.e., we assume that $s$ is given by 1.5 for some $k \in \mathbb{N}, \sigma_{1}, \sigma_{2} \in\{-1,1\}$. As above, Lemma 5(b) implies the equation

$$
4|\bar{a}(s)|^{2}-2 \zeta=4 s^{2}-2 \zeta \stackrel{1.5}{=} \frac{2}{3}\left(\zeta+4 d k^{2}-2 \sigma_{1} \sqrt{\left(\zeta+d k^{2}\right)^{2}-3}\right)
$$

which shows that condition (S) from Theorem 4 (iii) guarantees the simplicity of $\operatorname{ker}\left(\bar{F}_{a}(0, s)\right.$ ).

\subsection{Transversality condition.}

In the calculations related to the verification of the transversality condition we will use the following short-hand notations. In the context of Theorem 3 where $(t, k)$ is a solution of (1.4) we write

$$
a=\left(\hat{a}_{1}(t), \hat{a}_{2}(t)\right), \quad \zeta=\hat{\zeta}(t), \quad \dot{a}=\left(\frac{d \hat{a}_{1}}{d t}(t), \frac{d \hat{a}_{2}}{d t}(t)\right), \quad \dot{\zeta}=\frac{d \hat{\zeta}}{d t}(t)
$$

and in the context of Theorem 4 where $(s, k)$ is a solution of 1.5 we write

$$
a=\left(\bar{a}_{1}(s), \bar{a}_{2}(s)\right), \quad f=\bar{f}(s), \quad \dot{a}=\left(\frac{d \bar{a}_{1}}{d s}(s), \frac{d \bar{a}_{2}}{d s}(s)\right), \quad \dot{f}=\frac{d \bar{f}}{d s}(s) .
$$

Furthermore we will use

$$
\varphi(x):=\varphi_{k}(a)(x)=\alpha \cos (k x), \quad \varphi^{*}(x):=\varphi_{k}^{*}(a)(x)=\beta \cos (k x),
$$

where the vectors $\alpha=\alpha^{k}, \beta=\beta^{k} \in \mathbb{R}^{2}$ were defined in (4.7), (4.8). We have to check the transversality condition in the possible bifurcation points that we determined in Section 4.2 . Hence we may use (4.6), i.e.,

$$
\left(\zeta+d k^{2}\right)^{2}-4\left(\zeta+d k^{2}\right)|a|^{2}+1+3|a|^{4}=0 .
$$


In view of the formulas (4.7) and (4.8) we will have to investigate the following cases:

Case (1): $\left|a_{1} a_{2}\right| \neq \frac{1}{2}$ or $3 a_{1}^{2}+a_{2}^{2} \neq \zeta+d k^{2}$

Case (2): $a_{1} a_{2}=\frac{1}{2}$ and $3 a_{1}^{2}+a_{2}^{2}=\zeta+d k^{2}$

Case (3): $a_{1} a_{2}=-\frac{1}{2}$ and $3 a_{1}^{2}+a_{2}^{2}=\zeta+d k^{2}$

We set $c_{k}:=\int_{0}^{\pi} \cos (k x)^{2} d x$. In all three cases Proposition 8 yields the following formula for every constant $\psi \in \mathbb{R}^{2} \subset H$ :

$$
\begin{aligned}
T(\psi):=\left\langle G_{a a}(a, \zeta, f)[\varphi, \psi], \varphi^{*}\right\rangle_{H} & =\left\langle D G_{a a}(a, \zeta, f)[\varphi, \psi], \varphi^{*}\right\rangle_{L^{2}} \\
& =-\left\langle\left(\begin{array}{c}
\varphi^{T} M_{1}(a) \psi \\
\varphi^{T} M_{2}(a) \psi
\end{array}\right), \varphi^{*}\right\rangle_{L^{2}} \\
\stackrel{4.7}{=} & -\left\langle\left(\begin{array}{c}
\alpha^{T} M_{1}(a) \psi \\
\alpha^{T} M_{2}(a) \psi
\end{array}\right) \cos (k \cdot), \beta \cos (k \cdot)\right\rangle_{L^{2}} \\
& =-\left(\begin{array}{c}
\alpha^{T} M_{1}(a) \psi \\
\alpha^{T} M_{2}(a) \psi
\end{array}\right)^{T} \beta \int_{0}^{\pi} \cos (k x)^{2} d x \\
& =-2 c_{k} \alpha^{T}\left(\begin{array}{cc}
3 a_{1} \beta_{1}+a_{2} \beta_{2} & a_{1} \beta_{2}+a_{2} \beta_{1} \\
a_{1} \beta_{2}+a_{2} \beta_{1} & a_{1} \beta_{1}+3 a_{2} \beta_{2}
\end{array}\right) \psi
\end{aligned}
$$

Using (4.7), (4.8) and $\left(\zeta+d k^{2}\right)^{2}-4|a|^{2}\left(\zeta+d k^{2}\right)+1+3|a|^{4}=0$ we find in case (1)

$$
\begin{aligned}
T(\psi) & =-2 c_{k}\left(\begin{array}{c}
a_{1}\left(-3+6 a_{1}^{2} a_{2}^{2}-3 a_{2}^{4}+9 a_{1}^{4}+\left(2 a_{2}^{2}-6 a_{1}^{2}\right)\left(\zeta+d k^{2}\right)+\left(\zeta+d k^{2}\right)^{2}\right) \\
a_{2}\left(-1+15 a_{1}^{4}+3 a_{2}^{4}+18 a_{1}^{2} a_{2}^{2}-\left(14 a_{1}^{2}+6 a_{2}^{2}\right)\left(\zeta+d k^{2}\right)+3\left(\zeta+d k^{2}\right)^{2}\right)
\end{array}\right)^{T} \psi \\
& =-2 c_{k}\left(\begin{array}{c}
a_{1}\left(-4+6 a_{1}^{4}-6 a_{2}^{4}+\left(6 a_{2}^{2}-2 a_{1}^{2}\right)\left(\zeta+d k^{2}\right)\right) \\
a_{2}\left(-4+6 a_{1}^{4}-6 a_{2}^{4}+\left(6 a_{2}^{2}-2 a_{1}^{2}\right)\left(\zeta+d k^{2}\right)\right)
\end{array}\right)^{T} \psi \\
& =-4 c_{k}\left(-2+3 a_{1}^{4}-3 a_{2}^{4}+\left(3 a_{2}^{2}-a_{1}^{2}\right)\left(\zeta+d k^{2}\right)\right) a^{T} \psi .
\end{aligned}
$$

In case (2) one has $\alpha=\left(2\left(a_{2}^{2}-a_{1}^{2}\right),-2\right)^{T}, \beta=(-2,0)^{T}$ and using $a_{1} a_{2}=\frac{1}{2}$ one obtains

$$
T(\psi)=4 c_{k} \alpha^{T}\left(\begin{array}{cc}
3 a_{1} & a_{2} \\
a_{2} & a_{1}
\end{array}\right) \psi=4 c_{k}\left(\begin{array}{c}
2 a_{1}\left(3 a_{2}^{2}-3 a_{1}^{2}-\frac{a_{2}}{a_{1}}\right) \\
2 a_{2}\left(a_{2}^{2}-a_{1}^{2}-\frac{a_{1}}{a_{2}}\right)
\end{array}\right)^{T} \psi=-4 c_{k}\left(6 a_{1}^{2}-2 a_{2}^{2}\right) a^{T} \psi
$$

while in case (3) we may use $\alpha=(2,0)^{T}, \beta=\left(2\left(a_{2}^{2}-a_{1}^{2}\right), 2\right)^{T}$ and $a_{1} a_{2}=-\frac{1}{2}$ to get

$$
T(\psi)=-4 c_{k}\left(\begin{array}{c}
3 a_{1} \beta_{1}+a_{2} \beta_{2} \\
a_{1} \beta_{2}+a_{2} \beta_{1}
\end{array}\right) \psi=-4 c_{k}\left(\begin{array}{c}
2 a_{1}\left(3 a_{2}^{2}-3 a_{1}^{2}+\frac{a_{2}}{a_{1}}\right) \\
2 a_{2}\left(a_{2}^{2}-a_{1}^{2}+\frac{a_{1}}{a_{2}}\right)
\end{array}\right)^{T} \psi=-4 c_{k}\left(2 a_{2}^{2}-6 a_{1}^{2}\right) a^{T} \psi .
$$

Summarizing these calculations we find

$$
T(\psi)=-4 c_{k} a^{T} \psi \cdot \begin{cases}-2+3 a_{1}^{4}-3 a_{2}^{4}+\left(3 a_{2}^{2}-a_{1}^{2}\right)\left(\zeta+d k^{2}\right) & \text { in case }(1), \\ 6 a_{1}^{2}-2 a_{2}^{2} & \text { in case (2), } \\ 2 a_{2}^{2}-6 a_{1}^{2} & \text { in case (3). }\end{cases}
$$


In a similar way we obtain in case (1)

$$
\begin{aligned}
\left\langle\varphi, \varphi^{*}\right\rangle_{L^{2}} & =\alpha^{T} \beta \int_{0}^{\pi} \cos (k x)^{2} d x \\
& =c_{k}\left(-1+4 a_{1}^{2} a_{2}^{2}+\left(3 a_{1}^{2}+a_{2}^{2}-\zeta-d k^{2}\right)^{2}\right) \\
& =c_{k}\left(\left(\zeta+d k^{2}\right)^{2}-2\left(\zeta+d k^{2}\right)\left(3 a_{1}^{2}+a_{2}^{2}\right)-1+4 a_{1}^{2} a_{2}^{2}+\left(3 a_{1}^{2}+a_{2}^{2}\right)^{2}\right) .
\end{aligned}
$$

Using again $\left(\zeta+d k^{2}\right)^{2}-4|a|^{2}\left(\zeta+d k^{2}\right)+1+3|a|^{4}=0$ in case (1) and performing the corresponding calculations for the cases (2) and (3) we arrive at

$$
\left\langle\varphi, \varphi^{*}\right\rangle_{L^{2}}=2 c_{k} \begin{cases}\left(\zeta+d k^{2}\right)\left(-a_{1}^{2}+a_{2}^{2}\right)-1+3 a_{1}^{4}+2 a_{1}^{2} a_{2}^{2}-a_{2}^{4} & \text { in case }(1), \\ 2 a_{1}^{2}-2 a_{2}^{2} & \text { in case }(2), \\ 2 a_{2}^{2}-2 a_{1}^{2} & \text { in case }(3) .\end{cases}
$$

Now we are going to use the formulas $(4.12),(4.13)$ in the concrete settings of Theorem 3 and Theorem 4 .

In Theorem 3: Let $f \in \mathbb{R}$ and let $t \in(-1,1)$ satisfy equation $(1.4)$ for some $k \in \mathbb{N}$ and some $\sigma \in\{1,1\}$. Since $\dot{a} \in \mathbb{R}^{2} \subset H$ is a constant vector the formulas (4.12), (4.13) and Proposition 8 yield in case (1)

$$
\begin{aligned}
\left\langle\hat{F}_{a t}(0, t)[\varphi], \varphi^{*}\right\rangle_{H} \stackrel{[4.3)}{=}\left\langle G_{a a}(a, \zeta, f)[\varphi, \dot{a}], \varphi^{*}\right\rangle_{H}+\dot{\zeta}\left\langle G_{a \zeta}(a, \zeta, f)[\varphi], \varphi^{*}\right\rangle_{H} \\
\stackrel{4.12}{=}-4 c_{k} a^{T} \dot{a}\left(-2+3 a_{1}^{4}-3 a_{2}^{4}+\left(3 a_{2}^{2}-a_{1}^{2}\right)\left(\zeta+d k^{2}\right)\right)+\dot{\zeta}\left\langle D^{-1} \varphi_{,} \varphi^{*}\right\rangle_{H} \\
=-4 c_{k} a^{T} \dot{a}\left(-2+3 a_{1}^{4}-3 a_{2}^{4}+\left(3 a_{2}^{2}-a_{1}^{2}\right)\left(\zeta+d k^{2}\right)\right)+\dot{\zeta}\left\langle\varphi, \varphi^{*}\right\rangle_{L^{2}} \\
\stackrel{4.13)}{=} 2 c_{k}\left(-\left(-4+6 a_{1}^{4}-6 a_{2}^{4}+\left(6 a_{2}^{2}-2 a_{1}^{2}\right)\left(\zeta+d k^{2}\right)\right) a^{T} \dot{a}\right. \\
\left.+\dot{\zeta}\left(\left(\zeta+d k^{2}\right)\left(-a_{1}^{2}+a_{2}^{2}\right)-1+3 a_{1}^{4}+2 a_{1}^{2} a_{2}^{2}-a_{2}^{4}\right)\right) .
\end{aligned}
$$

We will now insert the trivial solution $\left(a_{1}, a_{2}, \zeta\right)=\left(\hat{a}_{1}(t), \hat{a}_{2}(t), \hat{\zeta}(t)\right)$ from Lemma 5 and use the identities

$$
a^{T} \dot{a}=\frac{d}{d t} \frac{|\hat{a}(t)|^{2}}{2}=\frac{d}{d t} \frac{f^{2}\left(1-t^{2}\right)}{2}=-f^{2} t, \quad \dot{\zeta}=-2 f^{2} t+\left(1-t^{2}\right)^{-3 / 2} .
$$

Notice also that the necessary condition 4.6 for bifurcation becomes

$$
\zeta+d k^{2}=2 f^{2}\left(1-t^{2}\right)-\sigma \sqrt{f^{4}\left(1-t^{2}\right)^{2}-1}
$$

so that 1.4 is proved. After a lengthy computation we obtain

$$
\begin{aligned}
\left\langle\hat{F}_{a t}(0, t)[\varphi], \varphi^{*}\right\rangle_{H}= & 2 c_{k}\left(4 f^{6} t^{3}\left(1-t^{2}\right)^{2}+f^{4}\left(1-t^{2}\right)^{1 / 2}-2 t f^{2}-\left(1-t^{2}\right)^{-3 / 2}\right. \\
& \left.-\sigma \sqrt{f^{4}\left(1-t^{2}\right)^{2}-1}\left(4 f^{4} t^{3}\left(1-t^{2}\right)+f^{2}\left(2 t^{2}-1\right)\left(1-t^{2}\right)^{-1 / 2}\right)\right)
\end{aligned}
$$


and hence the transversality condition (H2) from Theorem 7 is satisfied whenever the righthand side is nonzero, i.e. when condition (T) holds. In case (2) or (3) the transversality condition is always satisfied. Indeed, proceeding as above and using the explicit formulas

$$
a_{1}^{2}=\frac{\sqrt{1-t^{2}}}{2|t|}, \quad a_{2}^{2}=\frac{|t|}{2 \sqrt{1-t^{2}}}, \quad f^{2}=\frac{1}{2|t|\left(1-t^{2}\right)^{3 / 2}}
$$

from Lemma 5 (a) we find in case $(2)$, where $t<0$, that

$$
\begin{aligned}
\left\langle\hat{F}_{a t}(0, t)[\varphi], \varphi^{*}\right\rangle_{H} & =T(\dot{a})+\dot{\zeta}\left\langle\varphi, \varphi^{*}\right\rangle_{L^{2}} \\
& \stackrel{4.12}{=}-4 c_{k} a^{T} \dot{a}\left(6 a_{1}^{2}-2 a_{2}^{2}\right)+\dot{\zeta} \cdot 2 c_{k}\left(2 a_{1}^{2}-2 a_{2}^{2}\right) \\
& =4 c_{k}\left(f^{2} t\left(6 a_{1}^{2}-2 a_{2}^{2}\right)+\left(-2 f^{2} t+\left(1-t^{2}\right)^{-3 / 2}\right)\left(a_{1}^{2}-a_{2}^{2}\right)\right) \\
& =4 c_{k}\left(f^{2} t \cdot 4 a_{1}^{2}+\left(1-t^{2}\right)^{-3 / 2}\left(a_{1}^{2}-a_{2}^{2}\right)\right) \\
& =-\frac{2 c_{k}}{|t|\left(1-t^{2}\right)^{2}} \neq 0
\end{aligned}
$$

and similarly in case (3), where $t>0$, that

$$
\left\langle\hat{F}_{a t}(0, t)[\varphi], \varphi^{*}\right\rangle_{H}=+\frac{2 c_{k}}{|t|\left(1-t^{2}\right)^{2}} \neq 0 .
$$

In Theorem 4: Now let $\zeta \in \mathbb{R}$ and let $s$ be given by (1.5). Since $\dot{a} \in \mathbb{R}^{2} \subset H$ is a constant vector we get from 4.12 and Proposition 8 in case (1)

$$
\begin{aligned}
&\left\langle\bar{F}_{a s}(0, s)[\varphi], \varphi^{*}\right\rangle_{H} \stackrel{4.3]}{=}\left\langle G_{a a}(a, \zeta, f)[\varphi, \dot{a}], \varphi^{*}\right\rangle_{H}+\dot{f}\langle\underbrace{G_{a f}(a, \zeta, f)[\varphi]}_{=0}, \varphi^{*}\rangle_{H} \\
& \stackrel{4.12}{=}-4 c_{k} a^{T} \dot{a}\left(-2+3 a_{1}^{4}-3 a_{2}^{4}+\left(3 a_{2}^{2}-a_{1}^{2}\right)\left(\zeta+d k^{2}\right)\right) .
\end{aligned}
$$

Recalling

we obtain

$$
a^{T} \dot{a}=\frac{d}{d s} \frac{|\bar{a}(s)|^{2}}{2}=\frac{d}{d s} \frac{s^{2}}{2}=s
$$

$$
\left\langle\bar{F}_{a s}(0, s)[\varphi], \varphi^{*}\right\rangle_{H}=-4 c_{k} s\left(-2+3 a_{1}^{4}-3 a_{2}^{4}+\left(3 a_{2}^{2}-a_{1}^{2}\right)\left(\zeta+d k^{2}\right)\right) .
$$

Now we substitute $\left(a_{1}, a_{2}\right)=\left(\bar{a}_{1}(s), \bar{a}_{2}(s)\right)$ from Lemma 5 and the value $s$ from (1.5) of Theorem 4 to obtain after a lengthy calculation

$$
\begin{aligned}
\left\langle\bar{F}_{a s}(0, s)[\varphi], \varphi^{*}\right\rangle_{H}= & \frac{4 \sigma_{1} c_{k} s}{27\left(1+\left(s^{2}-\zeta\right)^{2}\right)} \sqrt{\left(\zeta+d k^{2}\right)^{2}-3}\left(2 \zeta+5 d k^{2}-4 \sigma_{1} \sqrt{\left(\zeta+d k^{2}\right)^{2}-3}\right) \\
& \cdot\left(4 \zeta+d k^{2}-2 \sigma_{1} \sqrt{\left(\zeta+d k^{2}\right)^{2}-3}\right) \underbrace{\left(\zeta+d k^{2}+\sigma_{1} \sqrt{\left(\zeta+d k^{2}\right)^{2}-3}\right)}_{\neq 0} .
\end{aligned}
$$

Hence, using $s \neq 0$ (from (4.6) we get $|s|=|a(s)|=|a| \geq 1$ ) we find that the transversality condition holds if and only if

$\zeta+d k^{2} \neq \sqrt{3}$ and $4 \zeta+d k^{2}-2 \sigma_{1} \sqrt{\left(\zeta+d k^{2}\right)^{2}-3} \neq 0$ and $2 \zeta+5 d k^{2}-4 \sigma_{1} \sqrt{\left(\zeta+d k^{2}\right)^{2}-3} \neq 0$ 
which is precisely assumption $(\mathrm{T})$ in Theorem 4 .

In case (2) and (3) we have $|T(\dot{a})|=4 c_{k}|s|\left|3 a_{1}^{2}-a_{2}^{2}\right|$. So let us identify those values of $a_{1}, a_{2}, s, \zeta, d$ and $k \in \mathbb{N}_{0}$ where

$$
a_{1}^{2} a_{2}^{2}=\frac{1}{4}, \quad 3 a_{1}^{2}=a_{2}^{2} \text { and } 3 a_{1}^{2}+a_{2}^{2}=\zeta+d k^{2} \geq \sqrt{3},
$$

i.e., the situations in case (2), (3) where transversality fails. Since in this case $a_{1}^{2}=1 / \sqrt{12}$, $a_{2}^{2}=3 / \sqrt{12}$ we see that $s^{2}=a_{1}^{2}+a_{2}^{2}=2 / \sqrt{3}$, i.e.,

$$
s= \pm \sqrt{\frac{2}{\sqrt{3}}} \text { and either } \zeta=-\frac{1}{\sqrt{3}} \text { or } \zeta=\frac{5}{\sqrt{3}} \text {. }
$$

In all of these cases $3 a_{1}^{2}+a_{2}^{2}=\sqrt{3}$, i.e, $d$ and $k$ must be such that $\zeta^{2}+d k^{2}=\sqrt{3}$ so that the necessary conditions of bifurcation is satisfied but transversality fails. However, this is already covered by condition (T) which excludes $\zeta+d k^{2}=\sqrt{3}$. This finishes part (iii) of Theorem 4 .

\section{IlluUstrations}

\subsection{The case $\zeta=0, d=0.1$.}

The table on the right shows some of the values of $f$ for which AUTO detects bifurcations from the trivial branch. It also shows the values of $f, k$ and $\sigma_{1}, \sigma_{2}$ for which Theorem 4 predicts bifurcation. In all cases the conditions $(\mathrm{S})$ and $(\mathrm{T})$ are satisfied.

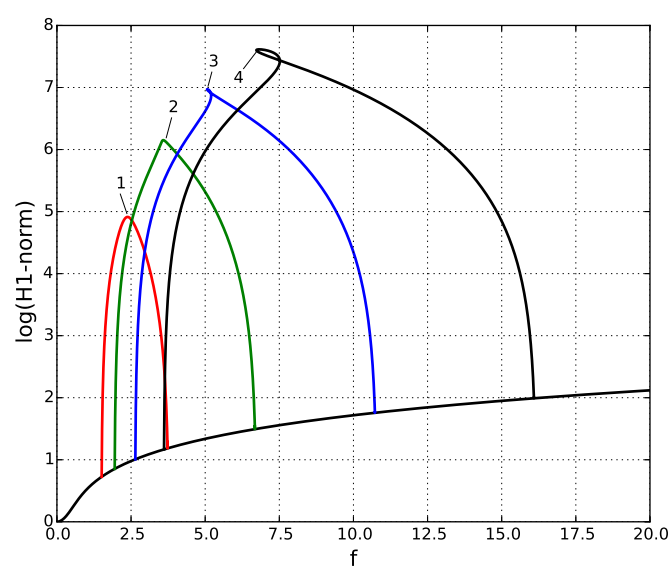

\begin{tabular}{|c|c|c|c|c|c|}
\hline curve & $f$ from AUTO & $f$ from Theorem 4 & $k$ & $\sigma_{1}$ & $\sigma_{2}$ \\
\hline red & 1.50873 & 1.508713281 & 5 & 1 & 1 \\
red & 3.73196 & 3.731954979 & 5 & -1 & 1 \\
green & 1.94874 & 1.948735656 & 6 & 1 & 1 \\
green & 6.67731 & 6.677307043 & 6 & -1 & 1 \\
blue & 2.64489 & 2.644940154 & 7 & 1 & 1 \\
blue & 10.7243 & 10.72430240 & 7 & -1 & 1 \\
black & 3.61248 & 3.612477695 & 8 & 1 & 1 \\
black & 16.0874 & 16.08735659 & 8 & -1 & 1 \\
\hline
\end{tabular}

FIGURE 1. Bifurcation points on trivial branch.

There are infinitely many branches bifurcating from the trivial one. Four different branches (red, green, blue, black) are shown in the bifurcation diagram. For graphical reasons $\log \left(\|a\|_{H^{1}}\right)$ is shown instead of $\|a\|_{H^{1}}$. We observe that all four depicted branches return to the trivial one. However, there is no proof of such a statement in general. The labels 1-4 on the four branches correspond to solutions with extremal norm. Their shapes are shown below

Figure 2. Bifurcation diagram 


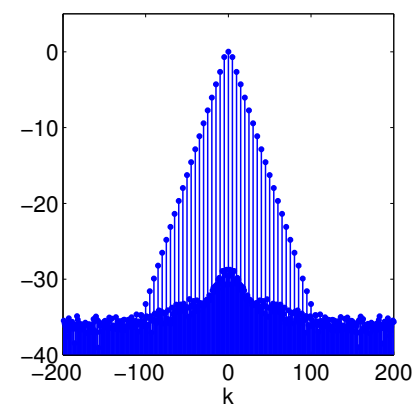

Label 1: $f=2.39993$

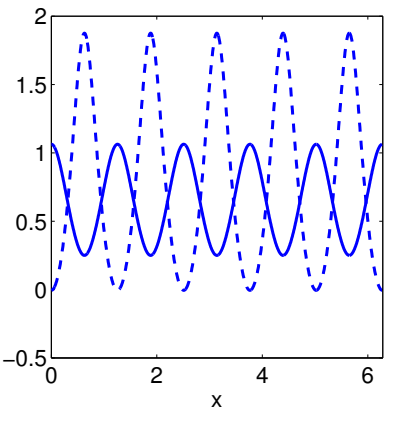

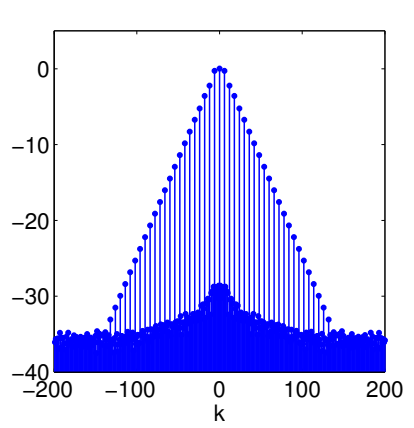

Label 2: $f=3.65501$

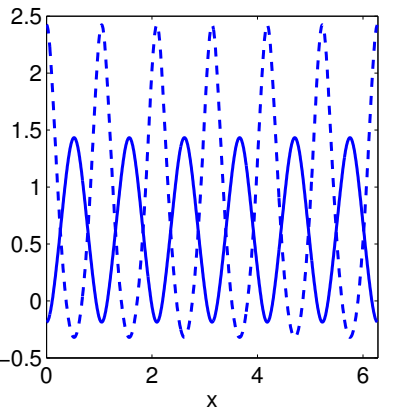

$a_{2}(x)$.
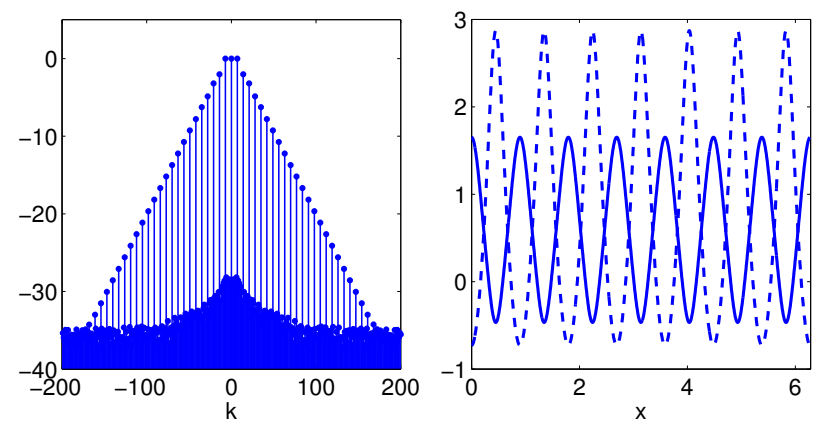

Label 3: $f=5.06244$
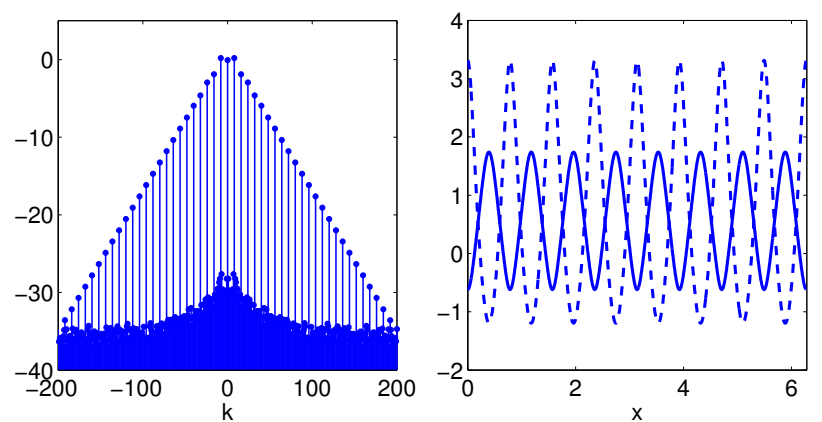

Label 4: $f=6.79129$

FigURE 4. Selected solutions. Left: $|\hat{a}(k)|$; right: solid $a_{1}(x)$, dashed $a_{2}(x)$.

\subsection{The case $\zeta=10, d=-0.2$.}

The table on the right shows all values of $f$ for which AUTO detects bifurcations from the trivial branch. It also shows the values of $f, k$ and $\sigma_{1}, \sigma_{2}$ for which Theorem 4 predicts bifurcation. In all cases the conditions $(\mathrm{S})$ and $(\mathrm{T})$ are satisfied. On two magenta colored branches solitons were found at turning points, cf. labels 1 and 2. Their shapes are shown below (on the left: $|\hat{a}(k)|$; on the right: $|a(x)|)$.

\begin{tabular}{|c|c|c|c|c|c|}
\hline curve & $f$ from AUTO & $f$ from Theorem 4 & $k$ & $\sigma_{1}$ & $\sigma_{2}$ \\
\hline red & 9.49912 & 9.499130565 & 6 & 1 & 1 \\
red & 12.0405 & 12.04059799 & 6 & -1 & 1 \\
green & 11.0296 & 11.02958498 & 5 & 1 & 1 \\
green & 11.5075 & 11.50749014 & 5 & -1 & 1 \\
blue & 11.8184 & 11.81841473 & 4 & 1 & 1 \\
blue & 8.87959 & 8.879591614 & 4 & -1 & 1 \\
black & 12.1610 & 12.16096908 & 3 & 1 & 1 \\
black & 6.02862 & 6.028624433 & 3 & -1 & 1 \\
magenta & 12.2805 & 12.28053072 & 2 & 1 & 1 \\
magenta & 3.97844 & 3.978438482 & 2 & -1 & 1 \\
magenta & 12.3071 & 12.30706762 & 1 & 1 & 1 \\
magenta & 3.21945 & 3.219445190 & 1 & -1 & 1 \\
\hline
\end{tabular}

FIGURE 5. Bifurcation points on trivial branch. 

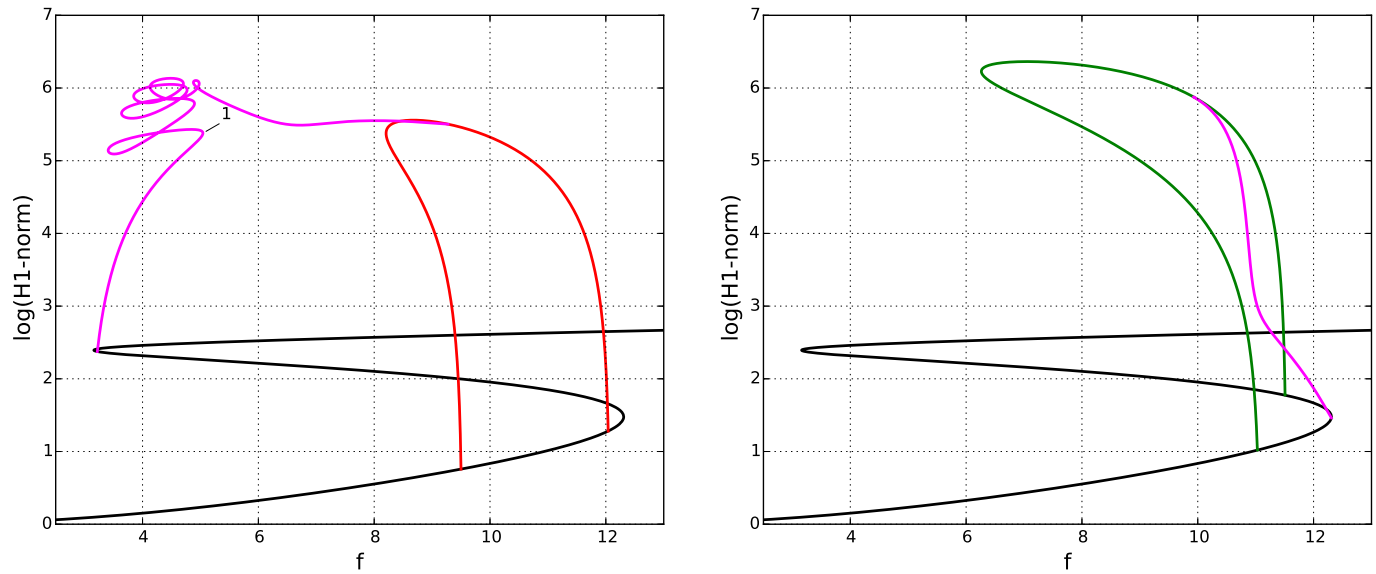

FiguRE 6. Bifurcation diagrams: red, green branch intersects twice, magenta branch once with trivial curve
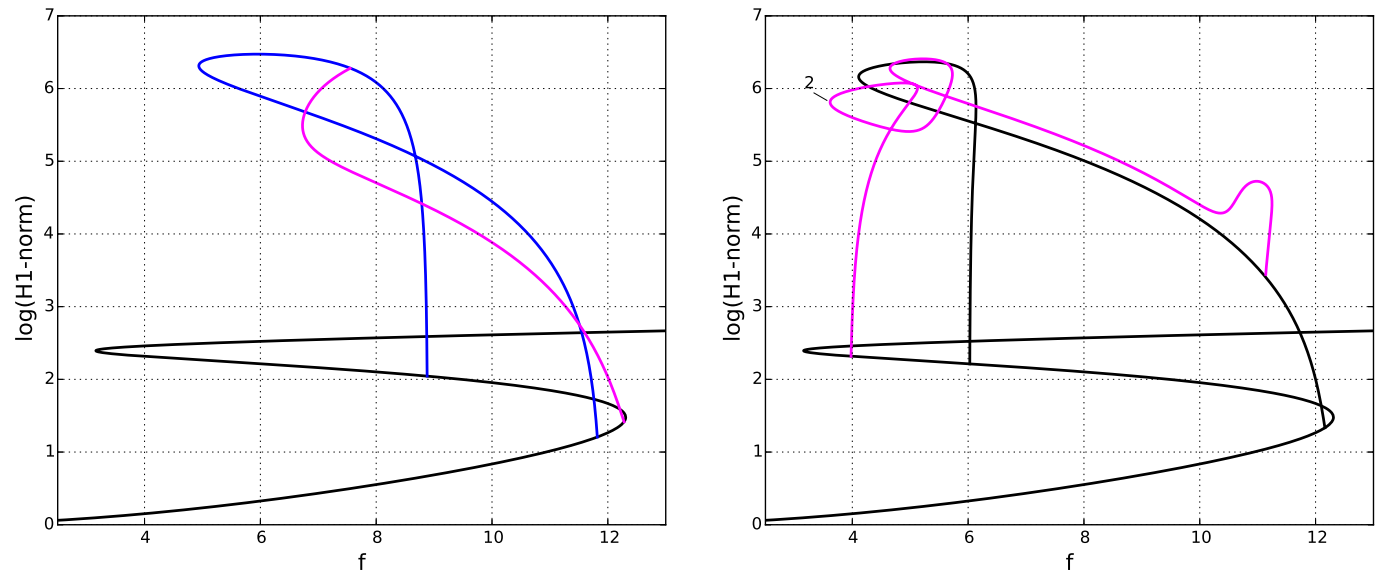

FiguRE 7. Bifurcation diagrams: blue, black branch intersects twice, magenta branch once with trivial curve
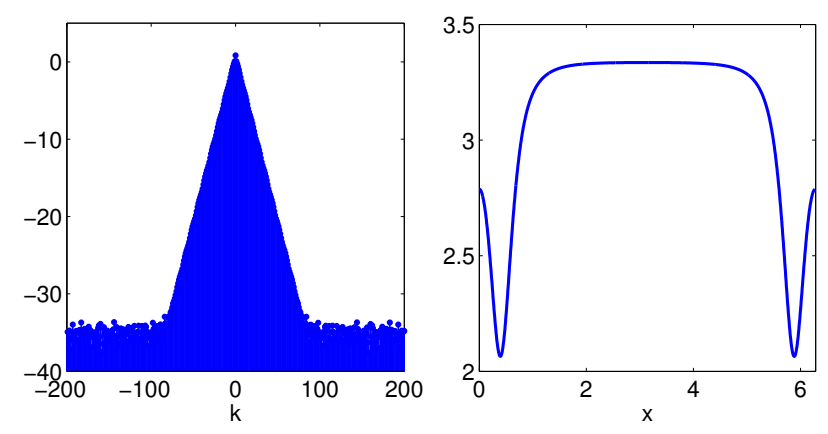

Label 1: dark 2-soliton at $f=5.04269$
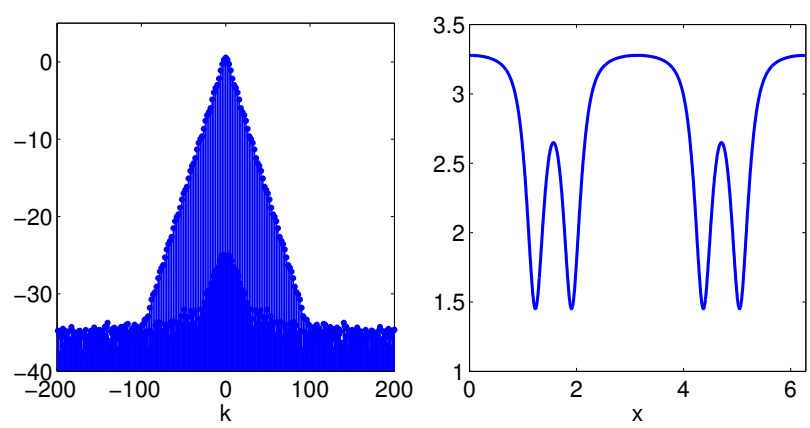

Label 2: dark 4-soliton at $f=3.61671$

Figure 8. Selected solutions. Left: $|\hat{a}(k)|$; right: $|a(x)|$. 
5.3. The case $f=1.6, d=0.1$.

The table on the right shows some of the values of $\zeta$ for which AUTO detects bifurcations from the trivial branch. It also shows the values of $\zeta$, $k$ and $\sigma$ for which Theorem 3 predicts bifurcation. In all cases the conditions $(\mathrm{S})$ and $(\mathrm{T})$ are satisfied.

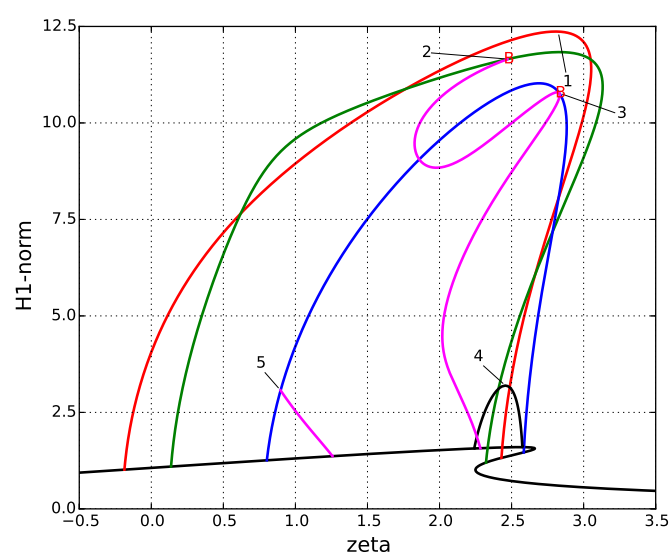

Figure 10. Bifurcation diagram

\begin{tabular}{|c|c|c|c|c|}
\hline curve & $\zeta$ from AUTO & $\zeta$ from Theorem 3 & $k$ & $\sigma$ \\
\hline red & -0.186656 & -0.186658434 & 5 & -1 \\
red & 2.42954 & 2.429546285 & 5 & -1 \\
green & 0.136823 & 0.13682209 & 4 & 1 \\
green & 2.32245 & 2.322478463 & 4 & -1 \\
blue & 0.80166 & 0.8016607862 & 6 & -1 \\
blue & 2.58448 & 2.584485791 & 6 & -1 \\
black & 2.24092 & 2.240849152 & 7 & -1 \\
black & 2.57475 & 2.574746606 & 7 & -1 \\
magenta & 1.25702 & 1.257021793 & 3 & 1 \\
magenta & 2.28327 & 2.283274503 & 2 & 1 \\
\hline
\end{tabular}

Figure 9. Bifurcation points on trivial branch.

The magenta branches are bifurcating from the trivial branch and connect at labels 2, 3, 5 to further branches. AUTO detects bifurcation points at labels 2 and 3. Label 5 is not detected by AUTO as a bifurcation point although the inspection of the solutions at the crossing suggests a true bifurcation. Labels 1 and 4 are selected as points with extremal norm.

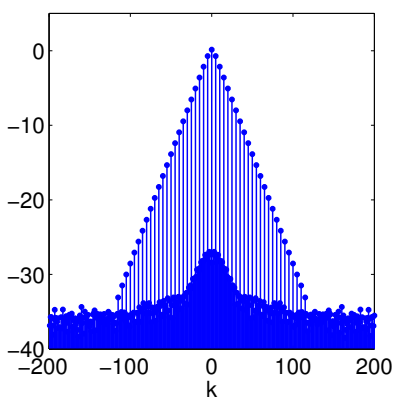

1: $\zeta=2.82318$

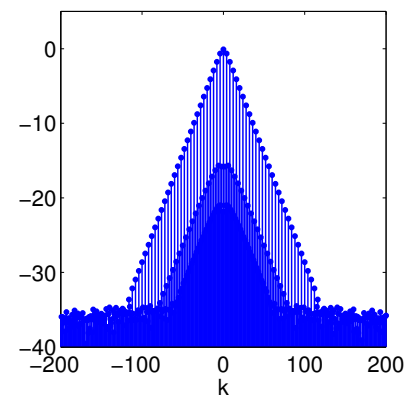

2: $\zeta=2.47286$

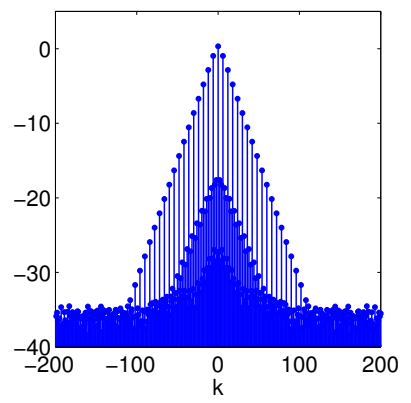

3: $\zeta=2.83117$

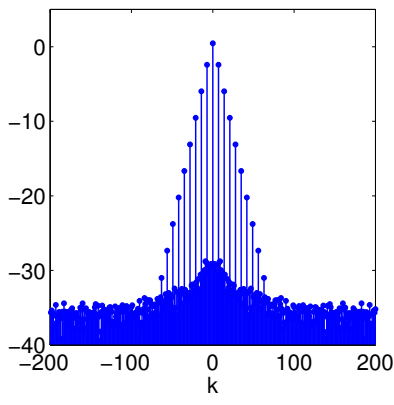

4: $\zeta=2.45756$

FIGURE 11. Fourier components $|\hat{a}(k)|$ of selected solutions. 
5.4. The case $f=2, d=-0.2$.

The table on the right shows some of the values of $\zeta$ for which AUTO detects bifurcations from the trivial branch. It also shows the values of $\zeta, k$ and $\sigma$ for which Theorem 3 predicts bifurcation. In all cases the conditions (S) and (T) are satisfied.

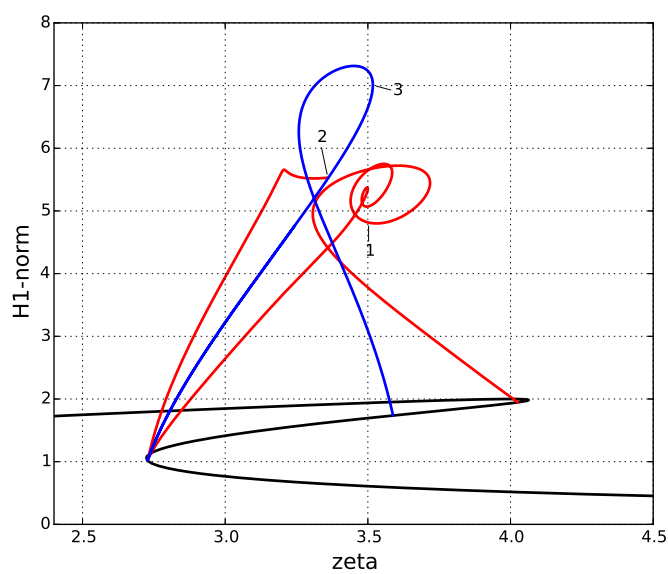

The branch bifurcating at $\zeta=2.79923$ is not depicted in the bifurcation diagram. The blue branch detaches from the trivial branch at $\zeta=$ 3.58827 and comes back to it at $\zeta=2.72872$. The red branch bifurcates from the trivial one at $\zeta=4.02617$. Apparently it does not come back to it but instead joins the blue branch at label 2 . At label 1 a 1-soliton was found and at label 3 a 2 -soliton.

Figure 13. Bifurcation diagram
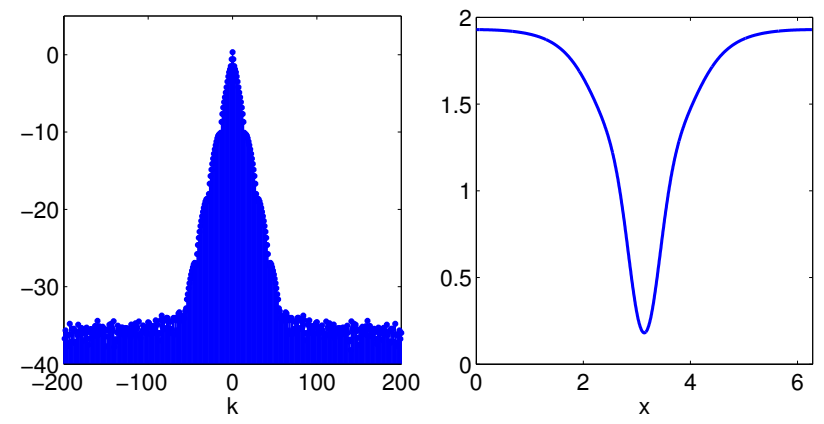

Label 1: dark 1-soliton at $\zeta=3.50256$
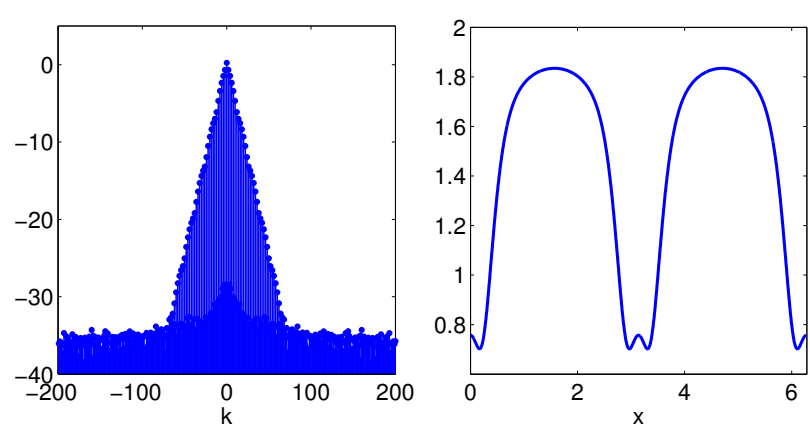

Label 3: dark 2-soliton at $\zeta=3.5175$

FIGURE 14. Selected bifurcation branches, selected solutions for $f=2, d=-0.2$.

\section{ACKNOWLEDGEMENTS}

Both authors thank J. Gärtner, T. Jahnke, Ch. Koos, P. Palomo-Marin, J. Pfeifle, and $\mathrm{Ph}$. Trocha (all from KIT) for fruitful discussions. We are grateful to T. Jahnke for letting us 
use his MATLAB code that postprocesses AUTO data and generates solution plots. Additionally, both authors gratefully acknowledge financial support by the Deutsche Forschungsgemeinschaft (DFG) through the research grant MA 6290/2-1 (first author) and CRC 1173 (second author).

\section{REFERENCES}

[1] A.R. Bishop, M.G. Froest, D.W. McLaughlin, and E.A. Overman. A modal representation of chaotic attractors for the driven, damped pendulum chain. Physical Letters A, 144:17-25, 1990.

[2] Y. K. Chembo and Nan Yu. Modal expansion approach to optical-frequency-comb generation with monolithic whispering-gallery-mode resonators. Physical Review A, 82:033801, 2010.

[3] Stéphane Coen and Miro Erkintalo. Universal scaling laws of Kerr frequency combs. Opt. Lett., 38(11):1790-1792, 2013.

[4] Michael G. Crandall and Paul H. Rabinowitz. Bifurcation from simple eigenvalues. J. Functional Analysis, 8:321-340, 1971.

[5] P. Del'Haye, A. Schliesser, O. Arcizet, T. Wilken, R. Holzwarth, and T.J. Kippenberg. Optical frequency comb generation from a monolithic microresonator. Nature, 450:1214-1217, 2007.

[6] Miro Erkintalo and Stéphane Coen. Coherence properties of Kerr frequency combs. Opt. Lett., 39(2):283286, 2014.

[7] Cyril Godey, Irina V. Balakireva, Aurélien Coillet, and Yanne K. Chembo. Stability analysis of the spatiotemporal Lugiato-Lefever model for Kerr optical frequency combs in the anomalous and normal dispersion regimes. Phys. Rev. A, 89:063814, 2014.

[8] G. Haller. Homoclinic jumping in the perturbed nonlinear Schrödinger equation. Comm. Pure Appl. Math., 52(1):1-47, 1999.

[9] T. Herr, V. Brasch, J. Jost, C.Y. Wang, N.M. Kondratiev, M.L. Gorodetsky, and T.J. Kippenberg. Temporal solitons in optical microresonators. Nature Photonics, 8:145-152, 2014.

[10] T. Herr, K. Hartinger, J. Riemensberger, C.Y. Wang, E. Gavartin, R. Holzwarth, M.L. Gorodetsky, and T.J. Kippenberg. Universal formation dynamics and noise of Kerr-frequency combs in microresonators. Nature Photonics, 6:480-487, 2012.

[11] T. Jahnke, M. Mikl, and R. Schnaubelt. Strang splitting for a semilinear Schrödinger equation with damping and forcing. CRC 1173-Preprint 2016/4, Karlsruhe Institute of Technology, 2016.

[12] Hansjörg Kielhöfer. Bifurcation theory, volume 156 of Applied Mathematical Sciences. Springer, New York, second edition, 2012. An introduction with applications to partial differential equations.

[13] T. J. Kippenberg, R. Holzwarth, and S. A. Diddams. Microresonator-based optical frequency combs. Science, 332:555-559, 2011.

[14] Stefan Krömer, Timothy J. Healey, and Hansjörg Kielhöfer. Bifurcation with a two-dimensional kernel. J. Differential Equations, 220(1):234-258, 2006.

[15] Ping Liu, Junping Shi, and Yuwen Wang. Bifurcation from a degenerate simple eigenvalue. J. Funct. Anal., 264(10):2269-2299, 2013.

[16] L. A. Lugiato and R. Lefever. Spatial dissipative structures in passive optical systems. Phys. Rev. Lett., 58:2209-2211, 1987.

[17] T. Miyaji, I. Ohnishi, and Y. Tsutsumi. Bifurcation analysis to the Lugiato-Lefever equation in one space dimension. Phys. D, 239(23-24):2066-2083, 2010.

[18] Pedro Parra-Rivas, Damià Gomila, François Leo, Stéphane Coen, and Lendert Gelens. Third-order chromatic dispersion stabilizes Kerr frequency combs. Opt. Lett., 39(10):2971-2974, 2014.

[19] Paul H. Rabinowitz. Some global results for nonlinear eigenvalue problems. J. Functional Analysis, 7:487-513, 1971.

[20] Shaofei Wang, Hairun Guo, Xuekun Bai, and Xianglong Zeng. Analysis of high-order dispersion on ultrabroadband microresonator-based frequency combs. arXiv, 1403.0183, 2014. 
[21] David Westreich. Bifurcation at double characteristic values. J. London Math. Soc. (2), 15(2):345-350, 1977.

R. MANDEL

Scuola Normale Superiore di Pisa,

I-56126 PisA, ItALY

E-mail address: Rainer.Mandel@sns.it

W. REICHEL

Institute for Analysis, Karlsruhe Institute of Technology (KIT),

D-76128 KARLSRUHE, GERMANY

E-mail address: wolfgang.reichel@kit.edu 